\title{
Administration of BMP2/7 in utero partially reverses Rubinstein-Taybi syndrome-like skeletal defects induced by Pdk1 or Cbp mutations in mice
}

\author{
Jae-Hyuck Shim, ${ }^{1}$ Matthew B. Greenblatt,, ${ }^{1,2}$ Anju Singh, ${ }^{1}$ Nicholas Brady, ${ }^{1}$ Dorothy Hu, ${ }^{1}$
} Rebecca Drapp, ${ }^{1}$ Wataru Ogawa, ${ }^{3}$ Masato Kasuga, ${ }^{4}$ Tetsuo Noda, ${ }^{5}$ Sang-Hwa Yang, ${ }^{6}$ Sang-Kyou Lee, ${ }^{6}$ Vivienne I. Rebel,7 and Laurie H. Glimcher ${ }^{1,2}$

\begin{abstract}
1Department of Immunology and Infectious Diseases, Harvard School of Public Health, Boston, Massachusetts, USA. 2Department of Medicine, Harvard Medical School, Boston, Massachusetts, USA. ${ }^{3}$ Department of Internal Medicine, Division of Diabetes and Endocrinology, Kobe University Graduate School of Medicine, Kobe, Japan. ${ }^{4}$ Research Institute, National Center for Global Health and Medicine, Shinjuku-ku, Tokyo, Japan.

${ }^{5}$ Department of Cell Biology, Cancer Institute, Tokyo, Japan. ${ }^{6}$ Department of Biotechnology and National Creative Research Initiatives Center for Inflammatory Response Modulation, Yonsei University, Seoul, Republic of Korea. ${ }^{7}$ Department of Cellular and Structural Biology, Greehey Children's Cancer Research Institute, University of Texas Health Science Center at San Antonio, San Antonio, Texas, USA.
\end{abstract}

\begin{abstract}
Mutations in the coactivator CREB-binding protein (CBP) are a major cause of the human skeletal dysplasia Rubinstein-Taybi syndrome (RTS); however, the mechanism by which these mutations affect skeletal mineralization and patterning is unknown. Here, we report the identification of 3-phosphoinositide-dependent kinase 1 (PDK1) as a key regulator of CBP activity and demonstrate that its functions map to both osteoprogenitor cells and mature osteoblasts. In osteoblasts, PDK1 activated the CREB/CBP complex, which in turn controlled runt-related transcription factor 2 (RUNX2) activation and expression of bone morphogenetic protein 2 (BMP2). These pathways also operated in vivo, as evidenced by recapitulation of RTS spectrum phenotypes with osteoblast-specific $P d k 1$ deletion in mice ( $P d k 1^{o s x}$ mice) and by the genetic interactions observed in mice heterozygous for both osteoblast-specific Pdk1 deletion and either Runx2 or Creb deletion. Finally, treatment of $P d k 1^{o s x}$ and $C b p^{+/-}$embryos with BMPs in utero partially reversed their skeletal anomalies at birth. These findings illustrate the in vivo function of the PDK1-AKT-CREB/CBP pathway in bone formation and provide proof of principle for in utero growth factor supplementation as a potential therapy for skeletal dysplasias.
\end{abstract}

\section{Introduction}

Rubinstein-Taybi syndrome (RTS) was first described in 1963 as a condition characterized by impaired intellectual function, broad thumbs and halluces, craniofacial dysmorphism, and frequent fractures $(1,2)$. Characteristic craniofacial defects include a short philtrum, micrognathia, a high arched palate, and dental defects such as talon cusps, enamel hypoplasia, and abnormalities in tooth number (3). Genetically, RTS is associated with microdeletions, inversions, and translocations involving chromosome $16 \mathrm{p} 13.3$ (4). Mapping of the $16 \mathrm{p} 13.3$ region in patients with RTS has identified heterozygous mutations in CREB-binding protein (CBP) that account for approximately $40 \%$ of RTS cases (5). Cbp $\mathrm{P}^{+/-}$ mice show a similar range of defects as those in RTS, including neurodevelopmental and behavioral defects; oligodactyly; extra, split, or asymmetric vertebrae; calvarial hypomineralization; and delayed ossification at several sites (6-9). However, despite these advances in understanding the genetic basis of RTS, the mechanism by which these defects arise is unclear, and we have little insight into which pathways might function upstream and downstream of CBP during development.

Authorship note: Jae-Hyuck Shim and Matthew B. Greenblatt contributed equally to this work.

Conflict of interest: Laurie H. Glimcher is on the board of directors and holds equity in Bristol-Myers Squibb pharmaceutical company.

Citation for this article: J Clin Invest. 2012;122(1):91-106. doi:10.1172/JCI59466.
CBP was originally identified as a protein that binds to the phosphorylated form of its partner, the c-AMP response elementbinding protein (CREB) (10). CBP functions as a CREB coactivator, and its histone acetyltransferase activity and the degree of interaction between CBP and CREB are critical determinants of the overall transcriptional activity of the CREB/CBP complex. This $\mathrm{CREB} / \mathrm{CBP}$ interaction is governed by various upstream kinases, such as PKA (10), AKT (11), p90RSK (12), and cyclin-dependent kinases (13) that phosphorylate CREB at S133. The consequences of disrupting CREB/CBP function are demonstrated by the skeletal and cognitive defects in RTS. Despite this, little is understood about how CREB/CBP activity is linked in osteoblasts to the anabolic cues that regulate skeletal mineralization and patterning.

In particular, one set of such cues, IGF-1 and insulin, have received attention as important anabolic regulators of skeletal development and mineralization during embryogenesis and adult life. IGF-1 evokes an increase in bone mass in response to physiologic growth cues, and circulating IGF-1 levels correlate with bone mass in humans and mice $(14,15)$. In mice, loss of both Igf1 and $I g f 2$ or $I g f 1 r$ deficiency result in delayed skeletogenesis during embryonic development (16). Recently, insulin receptor signaling has been shown to function in osteoblasts through its regulation of runt-related transcription factor 2 (RUNX2, also known as CBFA1) and osteocalcin, which is essential for whole-body glucose metabolism $(17,18)$. Looking downstream of these receptors, mice with a double deletion of Irs 1 and Irs 2 or Akt 1 and Akt2, key 
signaling components of the IGF-1 and insulin pathways, display delayed bone development (19-22).

One challenge to studying insulin/IGF-1 signaling pathways is that many key mediators, such as IRSs, PI3Ks, and AKTs, have several isoforms, complicating genetic studies. Signaling though IGF-1 and insulin receptors, IRSs, and PI3Ks converges at the kinase 3-phosphoinositide-dependent kinase 1 (PDK1), which has only a single isoform. PDK1 in turn activates AGC kinase family members, which themselves phosphorylate a wide range of substrates. Here we report that selective PDK1 deletion in either mature osteoblasts or in osteoprogenitor cells leads to a spectrum of phenotypes consistent with RTS. These defects map to the CREB/CBP complex via AKT, which in turn controls RUNX2 activation and bone morphogenetic protein 2 (BMP2) expression. These pathways operate in vivo, as evidenced by genetic interaction between $P d k 1^{f l /+} o s x$ and either Run $x 2^{+/-}$or $\mathrm{Creb}^{+/-}$alleles. Treatment of $\mathrm{Pdk} 1^{\text {osx }}$ or $\mathrm{Cbp} \mathrm{p}^{+/-}$ embryos in utero with recombinant human BMP2/7 (rhBMP2/7) partially reverses their craniofacial abnormalities upon birth, demonstrating both that BMP2 is a functionally important target of a PDK1-AKT-CREB/CBP signaling axis and that growth factor supplementation is a valid therapeutic approach for the correction of skeletal dysplasia phenotypes of RTS.

\section{Results}

$P D K 1$ is required for skeletogenesis during embryonic development. We first localized PDK1 expression in bone using immunohistochemistry (IHC). In the calvarium, PDK1 was expressed in osteoblasts along the osteogenic front, nasal bone, and zygomatic arch and by osteocytes embedded in the bone matrix (Supplemental Figure 1; supplemental material available online with this article; doi:10.1172/ JCI59466DS1). This signal was specific, as mice lacking PDK1 expression in osteoblasts (see below) showed no staining.

Since germline deletion of $P d k 1$ results in early embryonic lethality (23), we generated mice lacking PDK1 expression in osteoblasts by crossing mice carrying the PDK1 floxed allele with the osterixcre deleter strain $\left(P d k 1^{o s x}\right.$ mice $)(24,25)$. $P d k 1^{o s x}$ mice displayed multiple skeletal anomalies and died around P2 due to impaired feeding (Figure 1, A and B; Supplemental Figure 2A; and Supplemental Table 1). Pdk1 ${ }^{\text {ssx }}$ mice displayed several features characteristic of patients with RTS and murine models of RTS, such as hypomineralization of the frontal, nasal, and maxillary bones; a high arched palate; micrognathia; and a shortened philtrum due to midface hypoplasia (8). Additionally, Pdk1 $1^{o s x}$ mice displayed feeding difficulties and altered morphogenesis of the sphenoid bone in the base of the skull, a specific feature reported in some patients with RTS (26). These defects were apparent during embryonic development, as E16.5 and E18.5 Pdk1 $1^{\text {osx }}$ embryos had a substantial delay in ossification of the calvarium, ribs, vertebrae, and mandible, along with mildly delayed ossification of the femur (Figure 1C and Supplemental Figure 2, B-D). In addition to calvarial hypomineralization, $P d k 1^{\text {osx }}$ mice displayed hypoplasia of the clavicle, a feature often associated with reduced expression and/or activity of RUNX2, the master regulator of osteoblast differentiation (27), as well as spontaneous fractures, a feature of RTS (28) (Figure 1B, bottom). No significant abnormalities were observed in mice expressing the osterix-cre transgene alone (Osx mice; Supplemental Figure 4).

The early onset of skeletal anomalies in $P d k 1^{o s x}$ mice suggests that PDK1 plays an important role during embryonic skeletal development. To extend these observations, we generated mice lacking PDK1 expression in osteoprogenitor cells by crossing Pdk1 floxed allele mice with the dermo1-cre deleter strain ( $P d k 1^{d m 1}$ mice) that targets undifferentiated mesenchyme, including osteoblast and chondrocyte precursors (29). The resulting mutant mice showed shortening of both the axial and appendicular skeleton and a severe impairment in ossification of the skull, vertebrae, ribs, clavicle, and long bones (Figure 1D, Supplemental Figure 2E, and Supplemental Figure 3A). In addition to the craniofacial RTS features noted in $P d k 1^{o s x}$ mice, $P d k 1^{d m 1}$ mice displayed asymmetric alignment at the sternocostal junction, a feature of the $\mathrm{Cbp}^{+/-}$model of RTS (8). Histologic analysis of E18.5 Pdk1 $1^{d m 1}$ mice showed delayed ossification, with persistent unossified remnants of the primitive cartilage template in the diaphysis (Supplemental Figure 3B). Likewise, remodeling of the growth plate cartilage into bony trabeculae was delayed, with increased amounts of cartilage present in the metaphysis. Despite the reduction in the overall size of long bones in Pdk1 $1^{d m} 1$ mice, growth plate architecture was only minimally disrupted, with similarly sized hypertrophic and proliferative zones. Due to the severity of these defects, $P d k 1^{d m 1}$ mice died at birth due to respiratory failure. All of these abnormalities were more severe than those observed using osterix-cre that deletes after commitment to the osteoblast lineage, implying that PDK1 has additional functions in osteoprogenitors during embryonic development. However, we cannot exclude that deletion in chondrocytes or chondrocyte precursors contributes to the severity of the phenotype.

Recently, 2 populations of osteoprogenitors that give rise to bone tissue have been isolated from fetal bones (30). One population of the progenitor cells with surface markers CD45-Tie2$\mathrm{\alpha v}^{+} \mathrm{CD} 105^{+}$Thy 1.2- $\left(\mathrm{CD} 105^{+}\right.$Thy $\left.1^{-}\right)$produces bone through a cartilage intermediate (endochondral ossification), whereas a second population, CD45-Tie2 ${ }^{-} \alpha v^{+} \mathrm{CD}_{105}{ }^{+}$Thy $1.2^{+}\left(\mathrm{CD} 105^{+} \mathrm{Thy} 1^{+}\right)$, forms bone without a cartilage intermediate (intramembraneous ossification). We fractionated fetal bone cells from E17.5 $P d k 1^{d m 1}$ and $P d k 1^{o s x}$ embryos and littermate controls and analyzed the expression of these surface markers using flow cytometry (Figure 2A and Supplemental Figure 2F). Consistent with the reduction in skeletogenesis observed in E18.5 Pdk1 ${ }^{d m 1}$ embryos, absolute numbers of both populations of osteoprogenitors were reduced in $P d k 1^{d m} 1$ embryos, whereas $P d k 1^{\text {osx }}$ embryos showed only a very modest decrease in $\mathrm{CD}_{105^{+}} \mathrm{Thy}^{+}{ }^{+}$intramembranous precursors. Thus, by comparing the phenotypes of $P d k 1^{d m 1}$ and $P d k 1^{o s x}$ embryos and their corresponding levels of osteoprogenitors, we conclude that PDK1 likely functions both in the specification of osteoprogenitors and in later stages of osteoblast differentiation.

PDK1 is essential for osteoblast differentiation. We next examined expression of osteoblast-specific markers in the calvarium of $P d k 1^{o s x}$ mice. Consistent with a severe delay in skeletal mineralization of $P d k 1^{o s x}$ mice, expression of bone sialoprotein 2 (Bsp2), osteocalcin (Bglap, also known as Ocn), osterix (Osx), and type 1 collagen $\alpha$ (Col1a1) was all reduced. Likewise, in situ hybridization analysis of $P d k 1^{o s x}$ calvarium showed a substantial decrease in secreted phosphoprotein $1(O p n)$ and Ocn expression in the osteogenic front, nasal bone, and zygomatic arch (Figure 2, B and C, and Supplemental Figure 5A). In contrast with previous reports (31-34), transcript and protein levels of RUNX2 were unaffected both in vivo and in vitro in cultured Pdk $1^{\text {osx }}$ calvarial osteoblasts (CalvObs), arguing that any influence of PDK1 on RUNX2 activity must occur by influencing RUNX2 activity and not RUNX2 expression (Supplemental Figure 5B).

To directly probe the function of PDK1 in osteoblast differentiation, CalvObs were isolated from $P d k 1^{f l f l}$ neonates and transduced 
A
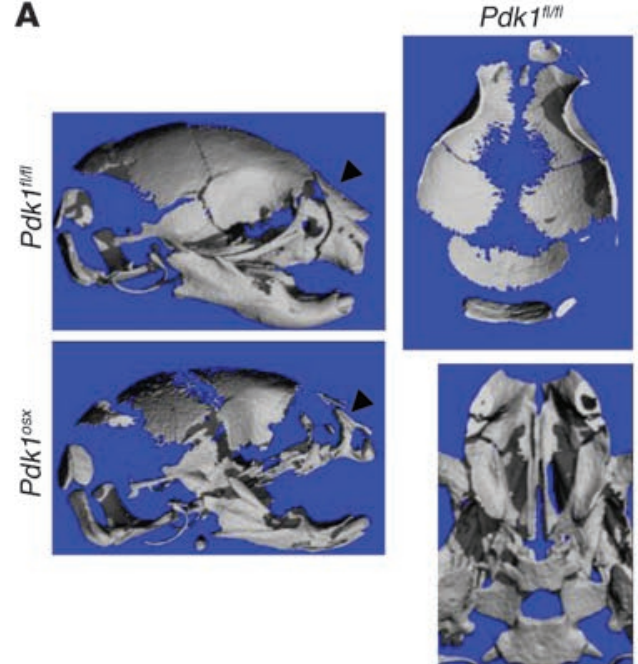

C $P d k 1^{\text {wan }}$
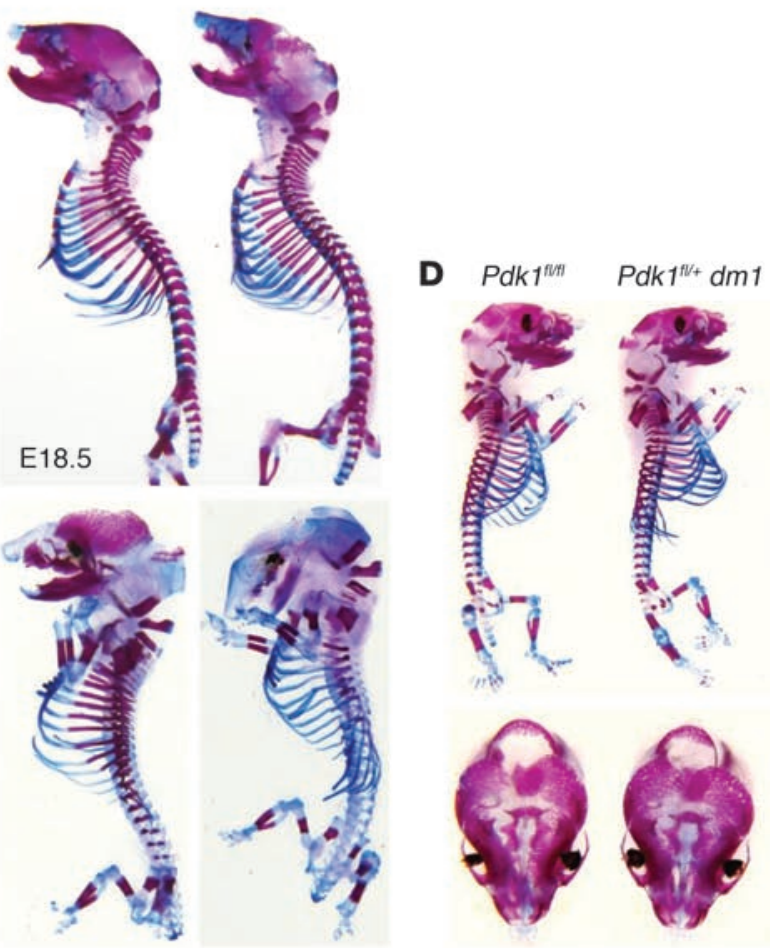

$P d k 1^{d m}$

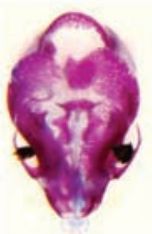

$P d k 1^{\text {osx }}$
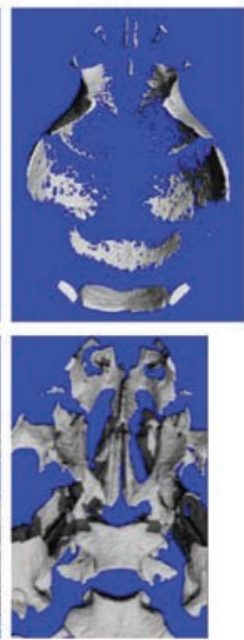

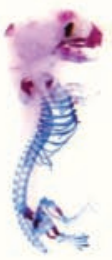

E18.5
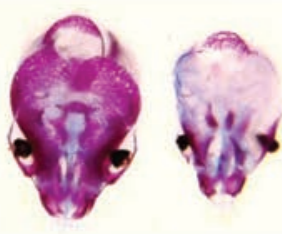

B
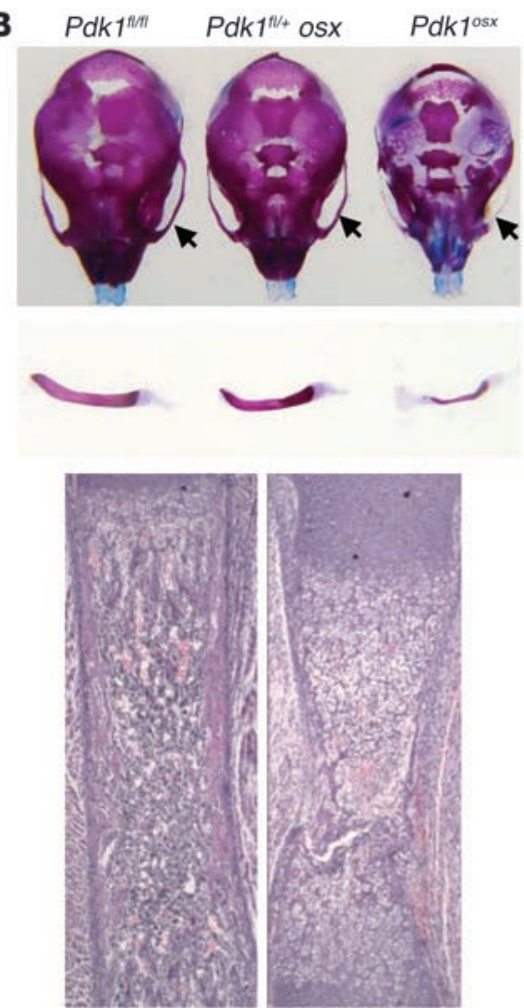

Pdk $1^{\text {thl }}$

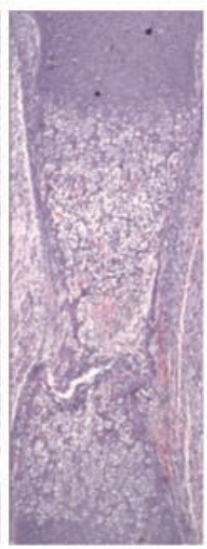

Pdk $1^{\text {osx }}$

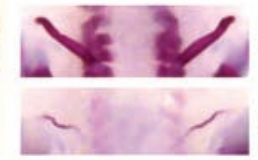

$P d k 1^{n+n}$

$P d k 1^{d m t}$

Figure 1

Abnormal skeletogenesis in PDK1-deficient mice. (A) Ossification of P2 Pdk $1^{f l l f l}$ and Pdk1osx skulls was analyzed by $\mu$ CT. Arrowheads indicate the nasal bone. (B) Alizarin Red/Alcian Blue-stained skeletal preps demonstrating calvarial hypomineralization and clavicular hypoplasia in P2 Pdk10sx mice. Arrows indicate the zygomatic bone (top). Hematoxylin and eosin-stained longitudinal section of the femur in P2 Pdk $1^{\text {fllfl }}$ and $P d k 1^{0 s x}$ mice (middle). Pdk10sx mice display low bone mass and spontaneous fractures (bottom). Original magnification, $\times 40$. (C and $\left.\mathbf{D}\right)$ Alizarin Red/Alcian Blue-stained skeletal preps of the indicated mice.

with lentiviruses expressing either EGFP control or cre recombinase. Efficient reduction of Pdk1 expression was confirmed by quantitative PCR and immunoblotting with an anti-PDK1 antibody (Supplemental Figure 5C). Lentiviral infection of CalvObs did not affect cell viability (Supplemental Figure 5D, top). Both cre-induced Pdk1 deletion and treatment with PI3K or PDK1 inhibitors blocked osteoblast differentiation, as determined by decreased alkaline phosphatase (ALP) activity and extracellular matrix mineralization (Figure 3A; Supplemental Figure 5D, bottom; and Supplemental Figure 6). Likewise, acquisition of the osteoblast differentiation markers $O s x, B s p 2$, Col1a1, and Ocn was reduced (Figure 3B). These defects required the kinase activity of PDK1, as the effect of PDK1 deletion could be rescued by enforced expression of WT but not kinase-dead PDK1 (Supplemental Fig- 

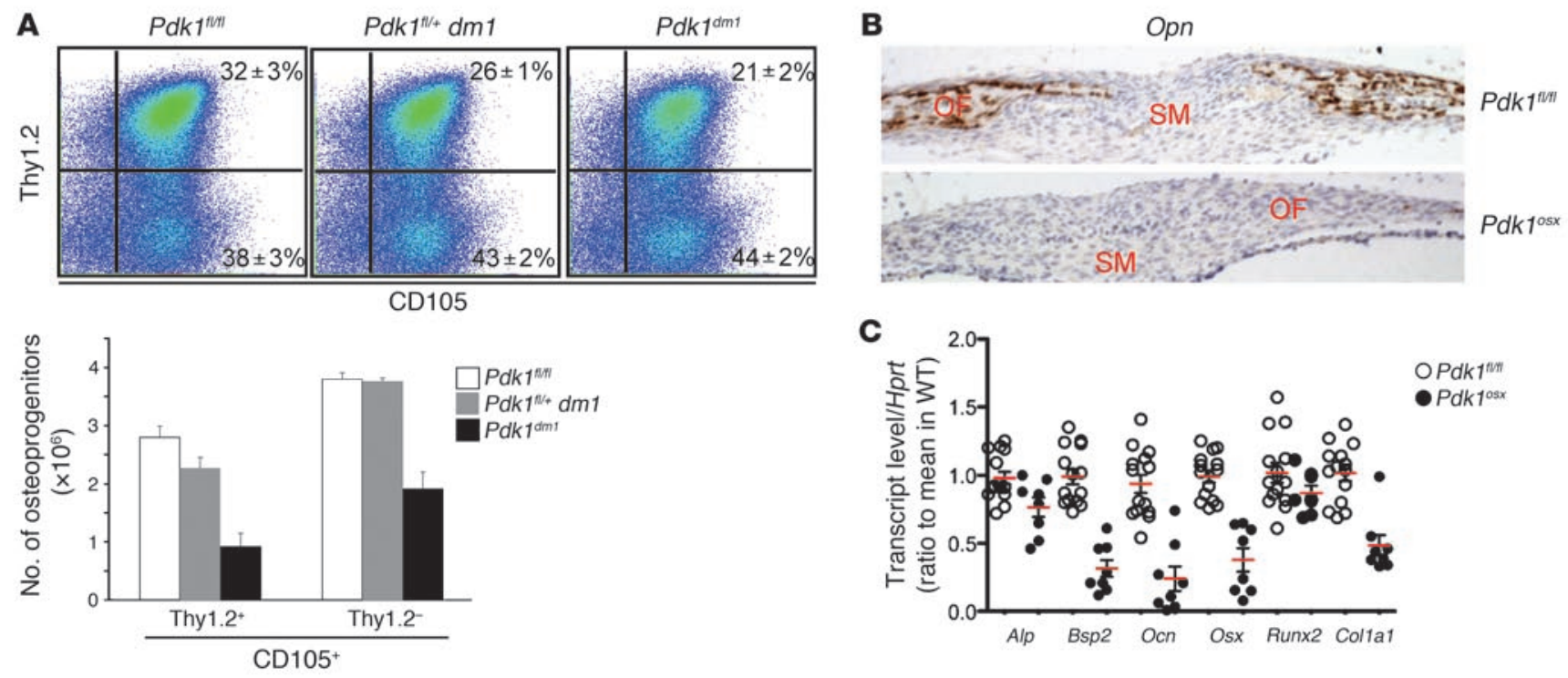

\section{Figure 2}

Characterization of osteoprogenitors and gene expression in Pdk10sx mice. (A) Representative FACS profiles on pregated, live CD45-Ter119- lineage cells harvested from long bones of E17.5 Pdk $1^{f l / f l}$ and $P d k 1^{d m 1}$ embryos. Percentages represent the percentage of cells within that quadrant. Values are mean \pm SD of biologic replicates. (B) Sections from the sagittal suture of P2 Pdk $1^{t / l f l}$ and $P d k 1^{\text {os } x}$ neonates analyzed for the expression of $O p n$ by in situ hybridization. Original magnification, $\times 100$. The osteogenic front (OF) and sutural mesenchyme (SM) are labeled. (C) Total RNA was extracted from calvaria of P2 Pdk $1^{f / f t}$ and $P d k 1^{\text {os } x}$ mice, and osteoblast gene expression was analyzed by quantitative PCR. The value of each sample, each from a different mouse, is indicated with a circle, and the average value of each group indicated with a red line.

ure 5, E and F). Together with skeletal anomalies in Pdk1 $1^{o s x}$ mice, these data confirm that PDK1 activity is essential for osteoblast differentiation and skeletal mineralization in vivo.

PDK1 is a key mediator of IGF-1 and insulin signaling in osteoblasts. In order to determine which pathways function upstream and downstream of PDK1 in osteoblasts, WT and PDK1-deficient CalvObs were stimulated with various ligands relevant to osteoblast function, including IGF-1, insulin, FGF-2, BMP2/7, TGF- $\beta$, and a WNT/Frizzled fusion protein. In the absence of PDK1, stimulation with IGF-1 failed to increase osteoblast differentiation, as shown by ablated induction of ALP activity, extracellular matrix mineralization, and osteoblast marker gene expression, whereas the responsiveness to FGF-2, BMP2/7, and TGF- $\beta$ was relatively normal in PDK1-deficient CalvObs (Figure 3, C and D, and Supplemental Figure 7). To probe the importance of PDK1 for the phosphorylation of AKT substrates, lysates from IGF-1-stimulated osteoblasts were blotted with an antibody recognizing the RXXS/T motif characteristic of AKT phosphorylation sites (Figure $4 \mathrm{~A}$ and ref. 35). IGF-1 stimulation failed to upregulate phosphorylation of AKT (T308) and several AKT substrates, including S6 (S235/236), in PDK1-deficient CalvObs (Figure 4A and Supplemental Figure 7F). Similar results were seen in WT CalvObs treated with PI3K, PDK1, or AKT inhibitors (Supplemental Figure 8A). AKT requires phosphorylation of both the PDK1-mediated T308 site and the S473 site, which is targeted by several other kinases $(35,36)$. Phosphorylation of AKT (S473), ERK1/2 and p38 MAPKs, and the mTOR target 4E-BP1 (T37/46) was unaffected or modestly increased in response to IGF-1, indicating that MAPK and mTOR signal transduction downstream of IGF-1 does not require PDK1. Glycogen synthase kinase-3 $\beta$ (GSK-3 $\beta$ ) showed a substantial decrease under basal conditions but only a slight defect after
IGF-1 stimulation (Supplemental Figure 7F). Further examination of Wnt/ $\beta$-Catenin signaling showed that PI3K or PKD1 inhibitors did not affect or slightly increased the ability of a Wnt/Frizzled fusion protein to activate the $\beta$-catenin-responsive reporter gene (Top flash-luc) (Supplemental Figure 7G). Similarly, protein levels of $\beta$-Catenin and basal Top flash reporter activity were minimally altered in PDK1-deficient CalvObs (Supplemental Figure 7H). Thus, PDK1 appears to play a selective role in regulation of the AKT pathway downstream of IGF-1 signaling.

CalvObs lacking PDK1 display similar defects in response to insulin (Figure 4B and Supplemental Figure 7F). As a sequela of impaired osteoblast responsiveness to insulin, $P d k 1^{o s x}$ mice displayed an increase in nonfasting serum glucose levels and a decrease in nonfasting serum insulin levels, a pattern previously observed with impaired insulin signal transduction in osteoblasts (Supplemental Figure 4D). Thus, PDK1 expression in osteoblasts contributes to systemic glucose metabolism $(17,37)$. All together, these results demonstrate that PDK1 selectively regulates the AKT pathway responses to IGF-1 and insulin but not to other osteoblast-activating stimuli, such as FGF-2, BMP2/7, TGF- $\beta$, or WNTs.

$P D K 1$ regulates $C R E B$ activity via $A K T$ in osteoblasts. To define the downstream targets of PDK1 in IGF-1 and insulin signaling pathways, we probed the activation of various signaling intermediates. In particular, AKT was considered, because it is a well-characterized PDK1 substrate (35) and its importance in osteoblast function has been previously described $(22,32-34)$. Likewise, treatment with an AKT inhibitor completely abolished basal and IGF-1-induced phosphorylation of AKT substrates, resulting in impaired osteoblast differentiation (Supplemental Figure 6A and Supplemental Figure 8A). As with other ACG family kinases, AKT requires phosphorylation at 2 separate sites for full activity - the T308 site, which 
A
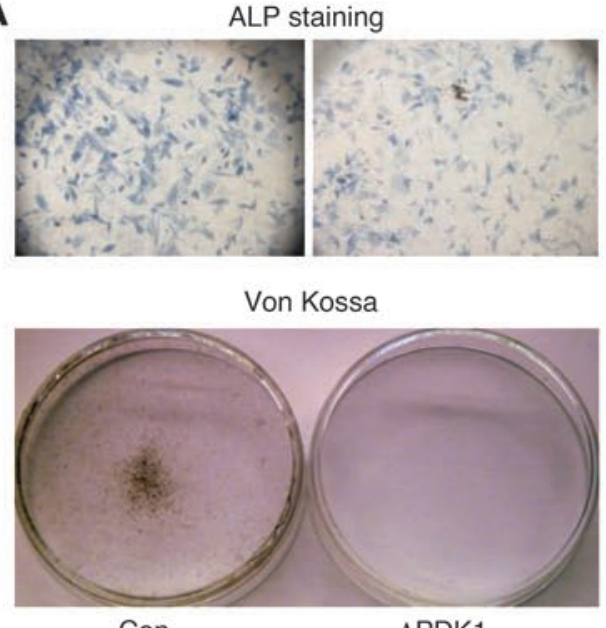

Con
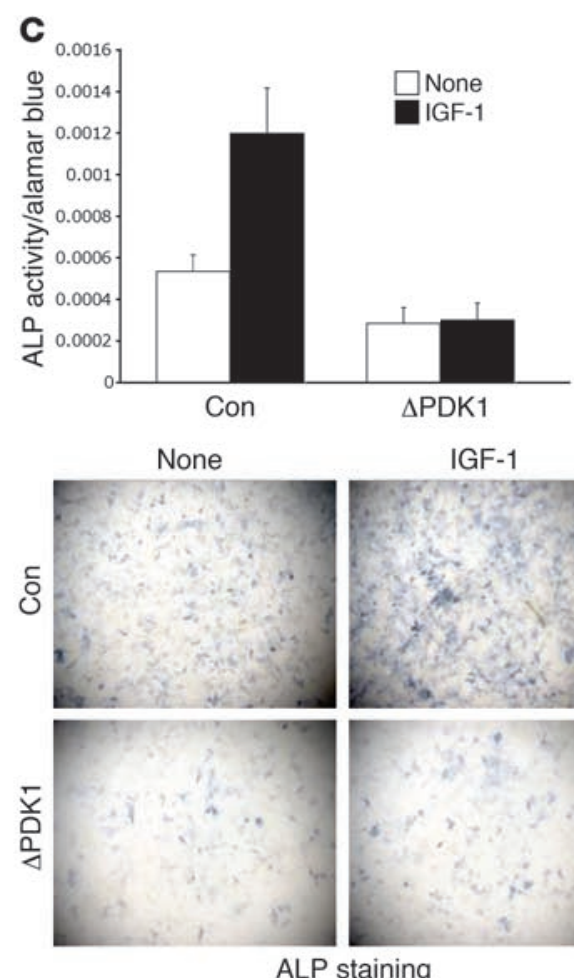

ALP staining
B
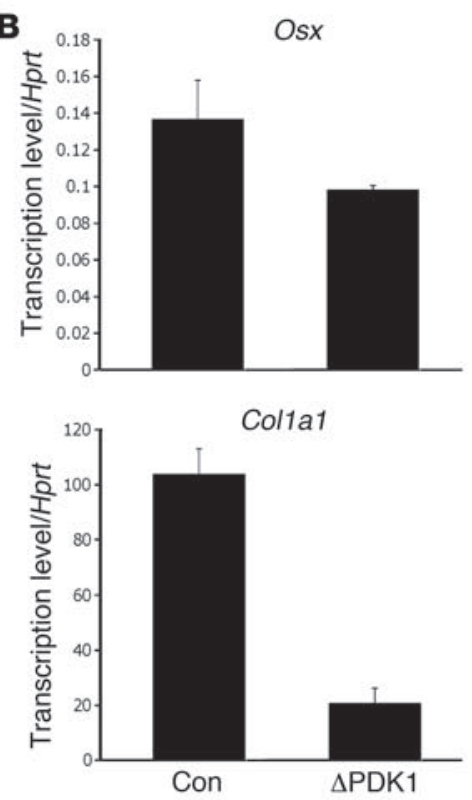

D
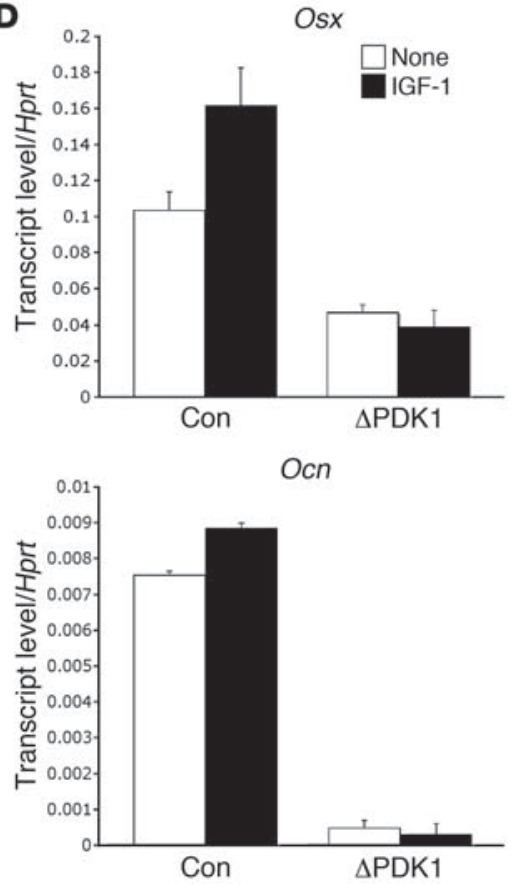
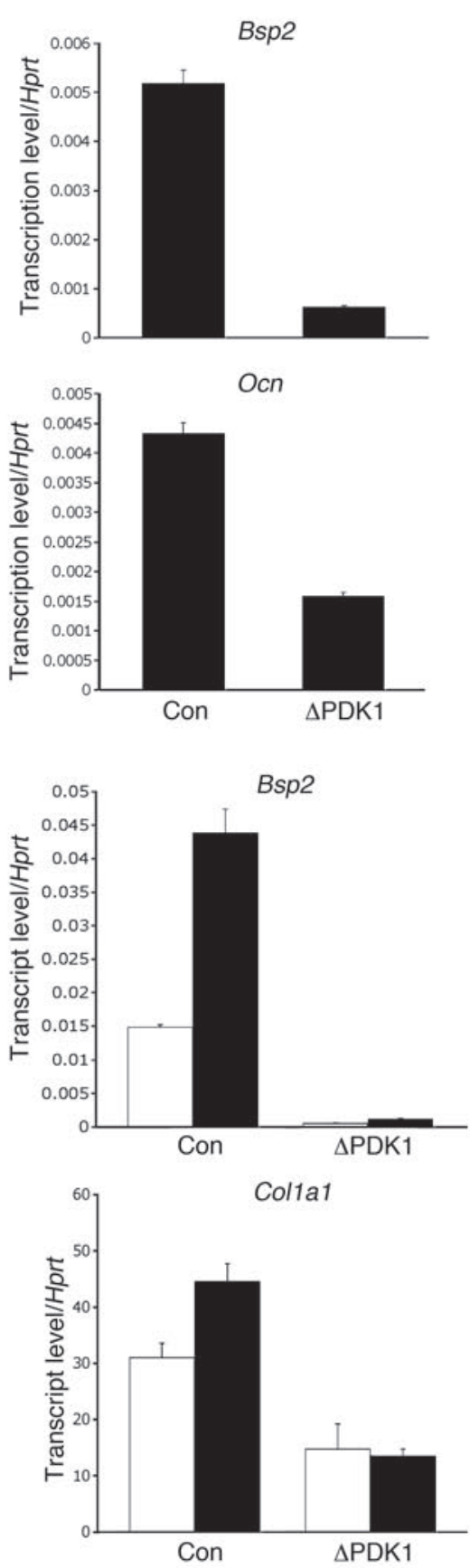

Figure 3

Characterization of PDK1-deficient osteoblasts. (A and B) Pdk1 $1^{f l f f}$ CalvObs infected by control (con) or Cre-expressing lentivirus ( $\left.\triangle \mathrm{PDK} 1\right)$ were cultured for 6 days and analyzed for ALP activity and Von Kossa staining or were analyzed by quantitative PCR. (C and D) Pdk $1^{f / / f l}$ CalvObs were infected with control or Cre-expressing lentivirus, cultured for 6 days in the absence or presence of IGF-1 (25 ng/ml), and (C) analyzed for ALP activity or (D) analyzed by quantitative PCR. Original magnification, $\times 100$.

is PDK1 mediated, and the S473 site, which is targeted by several other kinases, including mTOR and PDK2 $(35,36)$. In PDK1-deficient osteoblasts, levels of AKT phosphorylation at the T308 site were substantially reduced, whereas $\mathrm{S} 473$ phosphorylation was unaffected. Additional downstream targets examined, such as ERK, p38, and JNK MAPKs, showed unaltered or increased phosphorylation (Figure 5A). Surprisingly, despite the reduction in AKT T308 phosphorylation, the vast majority of AKT substrates were unaf- fected by the absence of PDK1. Only 3 bands at approximately 40 to $45 \mathrm{kDa}$ and $32 \mathrm{kDa}$ showed markedly decreased phosphorylation levels. Through published information of AKT substrates containing the RXXS/T* motif, these were independently identified though phospho-specific Western blotting to represent phosphoGSK-3 3 (S9), phospho-CREB (S133) $(11,38,39)$, and phospho-S6 (235/236), respectively (Figure 5, A and B, and refs. 11, 35, 40). This was confirmed in vivo by performing IHC analysis in the calvarium 
A

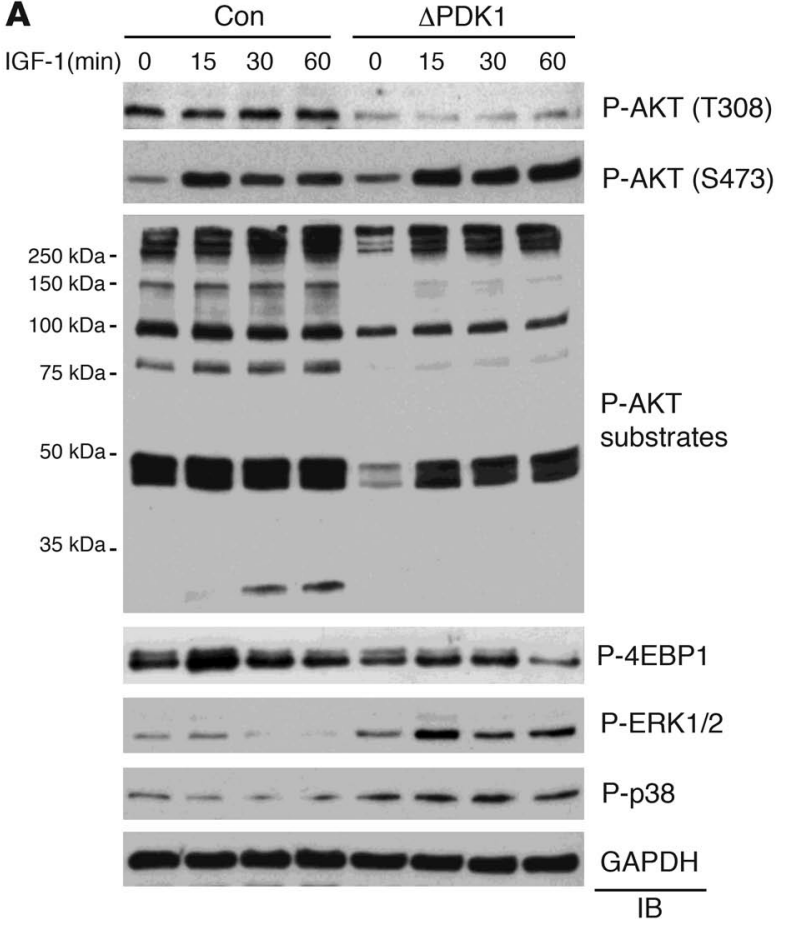

B

\begin{tabular}{|c|c|c|c|c|c|c|c|c|}
\hline $\mathbf{B}$ & \multicolumn{4}{|c|}{ Con } & \multicolumn{4}{|c|}{$\triangle \mathrm{PDK} 1$} \\
\hline sulin (min) & 0 & 15 & 30 & 60 & 0 & 15 & 30 & 60 \\
\hline
\end{tabular}

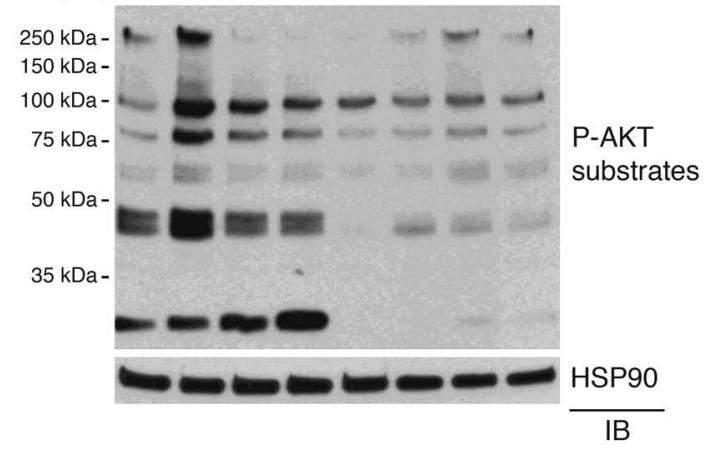

Figure 4

Altered responses to IGF-1 and insulin in osteoblasts lacking PDK1. Pdk 1/l/f CalvObs infected by control or Cre-expressing lentivirus, serum starved for 12 hours, and stimulated with (A) IGF-1 (25 ng/ml) or (B) insulin (10 nM). Cell lysates were immunoblotted with the indicated antibodies.

of $P d k 1^{o s x}$ and littermate control mice. Phosphorylation levels of CREB (S133), AKT (T308), GSK-3 $\beta$ (S9), and S6 (S235/236) were all decreased in osteoblasts lining the osteogenic fronts near the cranial sutures, nasal bones, and zygomatic arches (Figure 5B, right, and Supplemental Figure 8, B-E). Likewise, basal and IGF-1-induced phosphorylation of AKT substrates, including CREB, GSK-3 $\beta$, and S6, was all abolished in WT CalvObs treated with an AKT inhibitor (Supplemental Figure 8A and data not shown). Thus, the PDK1AKT signaling axis has a very specific role in regulating a small subset of AKT substrates in osteoblasts.

The CREB transcriptional factor has been shown to be a regulatory target for AKT, and its activity is modulated by AKTmediated phosphorylation at S133 $(11,38,39)$. We tested CREB transcriptional activity in PDK1-deficient CalvObs using the CREB-responsive luciferase reporter (CREB-Luc). Consistent with decreased CREB S133 phosphorylation in vivo and in vitro in the absence of PDK1, CREB activity was markedly reduced (Figure 5C). Likewise, IGF-1 stimulation failed to upregulate CREB S133 phosphorylation in the absence of PDK1 (Figure 5D). Insulin did not induce substantial CREB S133 phosphorylation, even in WT CalvObs (data not shown). Expression of Creb and Cbp was relatively unchanged in the absence of PDK1 (Supplemental Figure 9A). In PDK1-deficient CalvObs, CREB phosphorylation was normal or modestly increased in response to other CREB activators, such as FGF-2, isoproterenol (a $\beta_{2}$-adrenergic receptor agonist), and forskolin (a PKA agonist) (Supplemental Figure 9D). Therefore, $P d k 1$ deletion results in selective impairment in CREB phosphorylation downstream of IGF-1.

Another AKT substrate, S6, had reduced phosphorylation levels in vivo and in vitro (Supplemental Figure 7F and Supplemental
Figure 8, B-D). To assess whether this was functionally relevant, we used the observation that $\mathrm{S} 6$ phosphorylation is a critical regulator of cell size (41). Despite decreased S6 phosphorylation levels, cell size was unaltered in PDK1-deficient CalvObs (Supplemental Figure 9E). Thus, the decreased S6 phosphorylation levels do not alter function in PDK1-deficient osteoblasts, implying that other mechanisms may compensate for this defect.

To determine the physiologic contribution of CREB to bone formation in vivo, we examined the skeletal phenotype of Creb knockout mice. Since germline deletion of Creb results in perinatal lethality (42), we analyzed skeletal mineralization in embryonic $\mathrm{Creb}^{-/-}$and $\mathrm{Creb}^{+/+}$mice. E18.5 $\mathrm{Creb}^{-/-}$embryos displayed hypomineralization of the frontal, parietal, and maxillary bones and a mild delay in ossification of the femur (Figure 6, A and B). Unlike E18.5 Pdk $1^{\text {ssx }}$ and $C b p^{+/-}$embryos, gross mineralization of the axial skeleton and clavicle was relatively normal in the absence of CREB (Supplemental Figure 9B), which perhaps reflects compensatory effects from other CREB family members (43).

To determine whether there is a direct relationship between PDK1 and CREB activity in osteoblasts in vivo, we analyzed the genetic interaction between CREB and PDK1 by comparing WT, $\mathrm{Creb}^{+/-}$, and $P d k 1^{f l /+}$ os $x$ mice heterozygous for PDK1 and $P d k 1^{f l+} o s x$; $\mathrm{Creb}^{+/-}$double heterozygous mice. Dual heterozygosity for Creb and $P d k 1$ resulted in a substantial decrease in both femoral bone mass and calvarial mineralization beyond that seen with heterozygosity for either gene alone. The craniofacial and clavicular phenotypes were only minimally altered in $\mathrm{Pdk} 1^{\mathrm{fl/}+} \mathrm{osx} ; \mathrm{Creb}^{+/-}$double heterozygous mice (Figure 6C and Supplemental Figure 9C). Taken together, we conclude that PDK1 plays a key role in regulation of CREB activity via the AKT pathway in osteoblasts. 


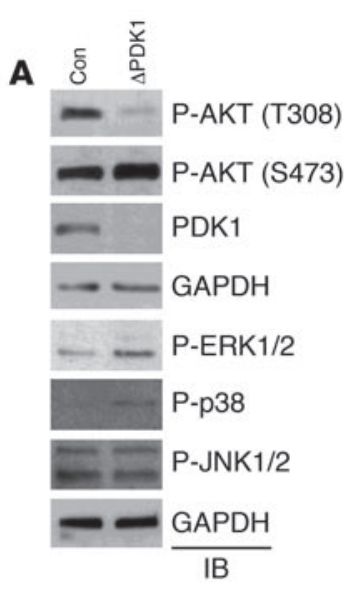

C

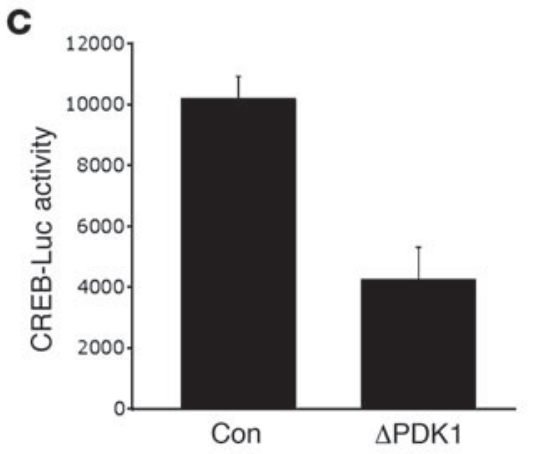

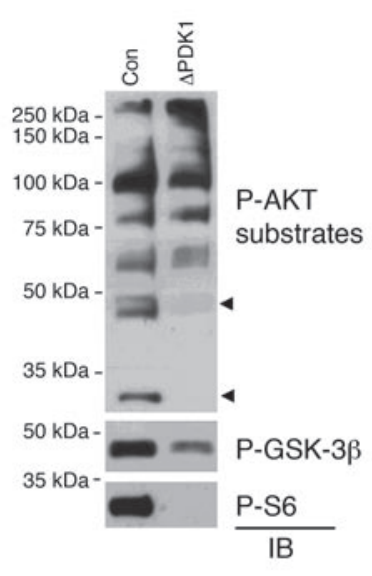

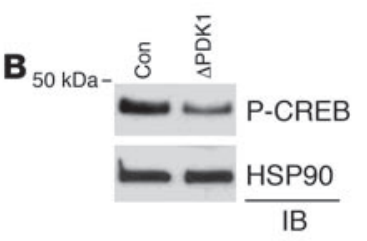

\section{P-CREB IHC}

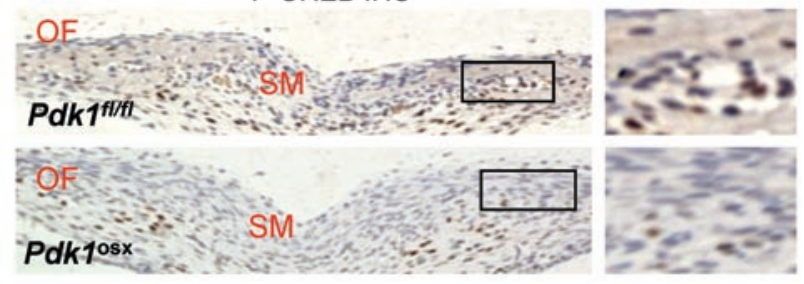

D

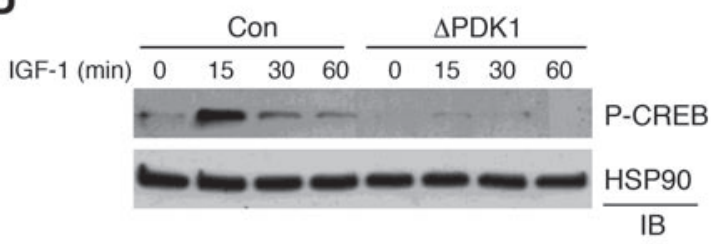

\section{Figure 5}

PDK1 activates CREB via AKT in osteoblasts. (A) Pdk $1^{f|l| f \mid}$ CalvObs were infected with control or Cre-expressing lentivirus, cultured for 6 days, and immunoblotted with the indicated antibodies. Arrowheads indicate predicted phosphorylated GSK-3 $\beta$ and S6 proteins. (B) Primary CalvObs were isolated from Pdk $1^{t / f f l}$ and $P d k 1^{o s x}$ neonates and immunoblotted with anti-phospho-CREB antibody. Alternatively, IHC was performed to show phosphorylation levels of CREB in a coronal section of the calvaria of P2 Pdk $1^{f l / f l}$ and Pdk 1 osx mice. Original magnification, $\times 100$. (C) Pdk $1^{f l / f l}$ CalvObs infected by control or Cre-expressing lentivirus were transfected with CREB-Luc and Renilla luciferase genes. Luciferase activity was analyzed 6 days after culture in differentiation medium. Results were normalized to a Renilla control. (D) Pdk $1^{f l / f l}$ CalvObs infected by control or Cre-expressing lentivirus were serum starved for 12 hours prior to IGF-1 stimulation $(25 \mathrm{ng} / \mathrm{ml})$. Cell lysates were then immunoblotted with anti-phospho-CREB (S133) antibody.

PDK1 regulates RUNX2 activity via the CREB/CBP complex. Previously, CBFA1/RUNX2 missense mutations were observed to have atypical manifestations, resulting in a syndrome sharing features of cleidocranial dysplasia (CCD) and RTS (44). Similarly, Pdk1 $1^{\text {osx }}$ mice display CCD phenotypes, such as clavicular hypoplasia and craniofacial hypomineralization. Pdk $1^{o s x}$ mice also display RTS phenotypes resembling Cbp haploinsufficiency (Figure 1). These findings suggest that PDK1 functions upstream of both RUNX2 and CBP in osteoblasts. To test this hypothesis, we first measured RUNX2 activity in PDK1-deficient CalvObs using the RUNX2responsive luciferase reporter (OSE2). As expected, RUNX2 activity was substantially reduced in the absence of PDK1, while the ATF4-responsive OSE1 luciferase reporter showed unaltered or modestly increased activity (Figure 7A). Accordingly, the defects in induction of ALP and osteoblast gene expression in PDK1-deficient osteoblasts could be partially rescued by enforced RUNX2 expression (Figure 7, B and C).

To extend the evidence that PDK1 regulates RUNX2 activity in osteoblasts from in vitro to in vivo systems, we analyzed the genetic interaction between RUNX2 and PDK1 by comparing WT, Run $x 2^{+/-}$, and $P d k 1^{f l+} o s x$ mice heterozygous for PDK1 and $P d k 1^{f l /+} o s x ; R u n x 2^{+/-}$ double heterozygous mice. Compound $P d k 1$ and Runx2 heterozy- gosity resulted in a substantial reduction in both femoral bone mass and calvarial hypomineralization beyond that seen with Pdk1 or Runx2 heterozygosity alone. Furthermore, the clavicular hypoplasia seen in $R u n \times 2^{+/-}$mice was further exacerbated by the addition of Pdk1 heterozygosity (Figure 8, A and B). Last, Runx2 and Pdk1 compound heterozygotes developed spontaneous rib fractures, providing functional evidence for the physiologic significance of this genetic interaction (Figure 8C). Thus, both in vivo and in vitro approaches confirm that PDK1 is a key regulator of RUNX2 transcriptional activity and, by extension, osteoblast function.

The above studies validate that PDK1 is required for activation of both RUNX2 and CREB, with CREB activation occurring via AKT $(11,39)$. To examine the relationship between CREB activation and RUNX2 activity in osteoblasts, we first tested the ability of CREB to induce RUNX2 activation using a RUNX2-responsive reporter (OSE2) (Figure 9A). Expression of a constitutively active mutant CREB, VP16/CREB (45), evoked an increase in RUNX2 activity, either alone or in combination with RUNX2 itself. Likewise, RUNX2 DNA-binding affinity was increased in the presence of CBP and VP16/CREB but not A-CREB, a dominant-negative CREB mutant (Figure 9B and ref. 46). VP16/CREB overexpression increased the interaction of RUNX2 with CBP in HEK293 cells (Figure 9C), where- 

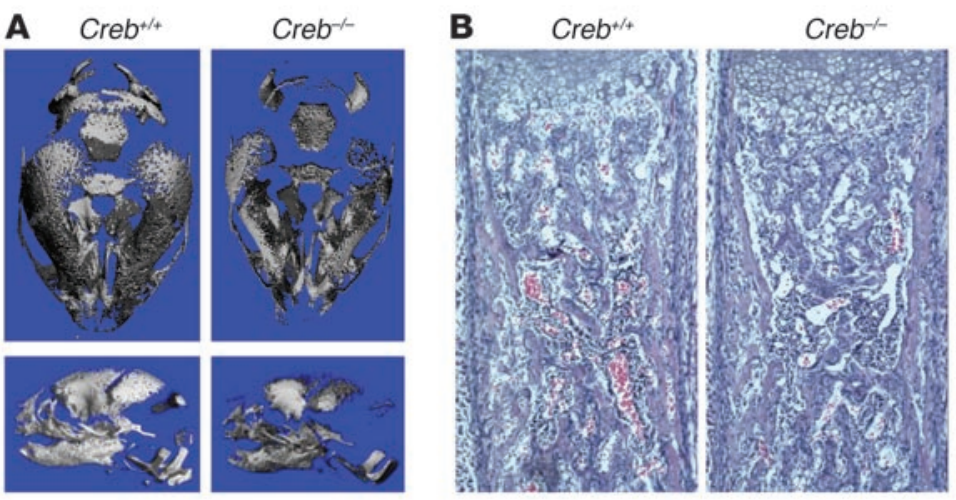

\section{Figure 6}

Characterization of skeletal defects in $\mathrm{Creb}^{-/-}$mice. (A) Ossification of E18.5 $\mathrm{Creb}^{+/+}$and $\mathrm{Creb}^{-/-}$skulls was analyzed by $\mu \mathrm{CT}$, and the 3D reconstruction is displayed. (B) Hematoxylin and eosin-stained longitudinal section of the femur in E18.5 $\mathrm{Creb}^{+/+}$and $\mathrm{Creb}^{-/-}$embryos. Original magnification, $\times 100$. (C) $\mu \mathrm{CT}$ analysis of the femurs from 4-week-old $P d k 1^{f l /+}$, $P d k 1^{f l /+o s x}, \mathrm{Creb}^{+/}$, and Pdk1 $1^{\mathrm{fl}+\mathrm{O}} \mathrm{Osx} ; \mathrm{Creb}^{+/-}$mice. BV/TV, bone volume fraction; Tb.Th, trabecular thickness; Tb.N, trabecular number; C.Th, cortical thickness. ${ }^{* *} P<0.005$, Student's $t$ test.
C

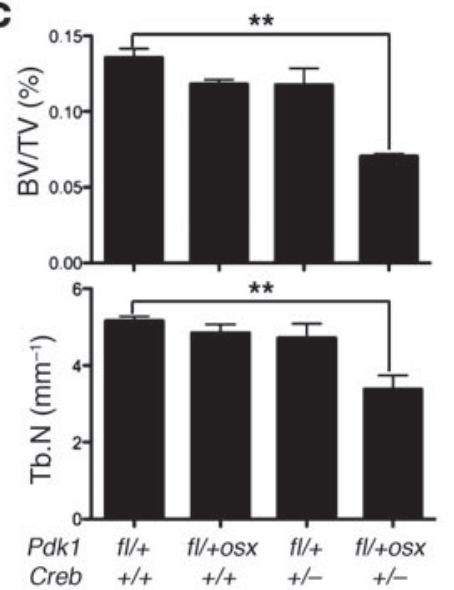

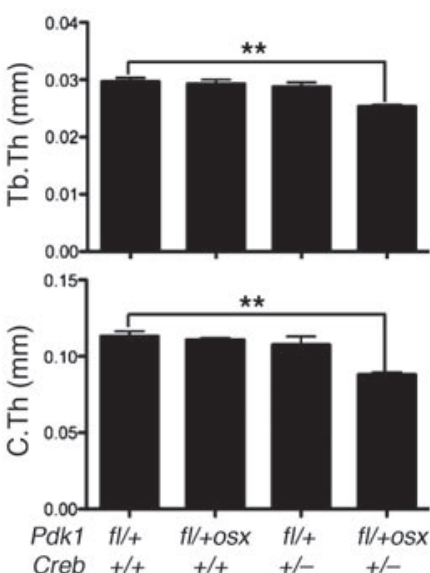

as in the RUNX2/CBP interaction, CREB phosphorylation, and transcriptional activity were all decreased in the absence of PDK1 (Figure 5, B and C, Figure 9D). Therefore, PDK1-mediated CREB activation can promote RUNX2 interaction with CBP in osteoblasts, thereby increasing RUNX2 transcriptional activity.

CREB transactivates BMP2 expression in osteoblasts. Spontaneous bone fractures observed in $P d k 1^{o s x}$ neonates and compound $P d k 1$ and Runx2 heterozygotes resemble the fractures resulting from limb-specific deletion of BMP2 (47). Additionally, recent in vitro analyses have suggested that BMP2 is a target of activated CREB in osteoblasts (48). To explore whether the PDK1 pathway is linked to the regulation of $B m p 2$ expression, human mesenchymal stem cells (hMSCs) or CalvObs were cultured under osteoblast differentiation conditions and treated with either PDK1 or PI3K inhibitors (Supplemental Figure 10A). Treatment with these inhibitors suppressed expression of Bmp2 in both systems. Similarly, Bmp2 transcript levels were markedly reduced in $P d k 1^{o s x}$ calvaria and PDK1-deficient CalvObs (Figure 10A, left, and Supplemental Figure 10B). Immunoblotting and IHC for phosphorylation of the BMP-responsive SMAD1/5/8 demonstrated a substantial reduction both in vitro and in vivo in the absence of PDK1 (Figure 10B). Likewise, Bmp2 transcript levels were reduced in $\mathrm{Creb}^{-/-}$calvaria (Figure 10A). Taken together, these results suggest that PDK1 acts through CREB to mediate Bmp2 transactivation in osteoblasts. To test this further, we examined the ability of CREB to induce $B m p 2$ expression using a luciferase reporter containing the distal and proximal promoter of the murine Bmp2 gene, BMP2-Luc $(-2712 /+165$ bp) (ref. 49 and Figure 10C). Luciferase activity showed a dose-dependent increase with expression of either WT
CREB or the constitutively active mutant VP16/CREB (Supplemental Figure 10C, top, and ref. 50). In addition, CREB activity was further increased in the presence of CBP, suggesting that CREB functions along with its coactivator CBP to upregulate Bmp2 expression (Supplemental Figure 10C, bottom). To identify the CREB-responsive elements (CREs) in the murine $B m p 2$ promoter, we compared CREB-induced luciferase activation of the extended (-2712/+165 bp) and proximal (-150/+165 bp) promoter constructs (Supplemental Figure 10D and ref. 49). Induction of CREB-mediated luciferase activation was similar, indicating that the CRE sites are localized in the proximal promoter. Two putative CRE sites in the proximal promoter at $-66 / 62$ bp and $+38 / 42$ bp were mutated in every combination of single and double mutants (Figure 10D). Mutation of the $-66 / 62$ bp site (mut 1 ) or both sites (-66/62 bp and $+38 / 42$ bp, mut 1 and mut 2 ) abolished responsiveness to WT CREB and VP16/CREB. Mutation of the $+38 / 42$ bp site alone did not affect CREB responsiveness, localizing the functionally relevant CRE site to $-66 / 62 \mathrm{bp}$. Accordingly, biotinylated oligonucleotides containing the WT but not the mutated $-66 / 62$ bp CRE site were able to pull-down CREB (Figure 10E). Thus, CREB transactivates $B m p 2$ by directly interacting with the CRE site (-66/62 bp) in the Bmp2 proximal promoter. We conclude that the CREB/CBP complex increases RUNX2 activity, both by promoting RUNX2 DNA binding and by directly transactivating Bmp2 expression through binding the $B m p 2$ promoter (see schematic below). rhBMP2/7 partially reverses the skeletal defects in $\mathrm{Pdk} 1^{\text {osx }}$ and $\mathrm{Cbp^{+/- }}$ embryos. rhBMP2 induces ectopic formation of bone and cartilage in vivo, osteogenic differentiation of mesenchymal stem cells to mature osteoblasts in vitro, and reverses bone loss in aged mice 
A
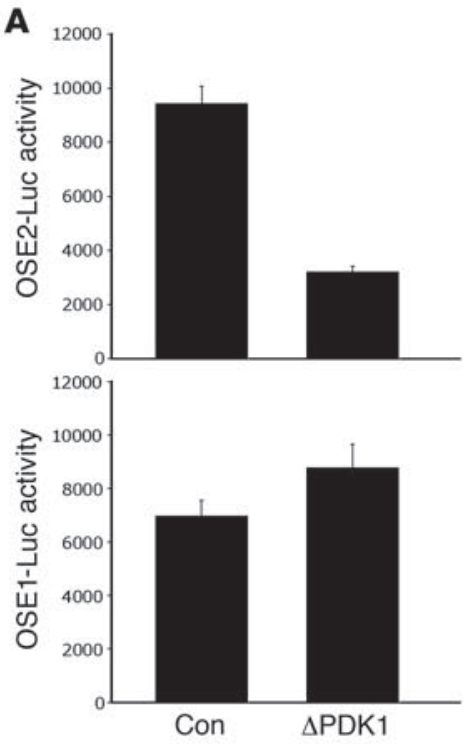

B
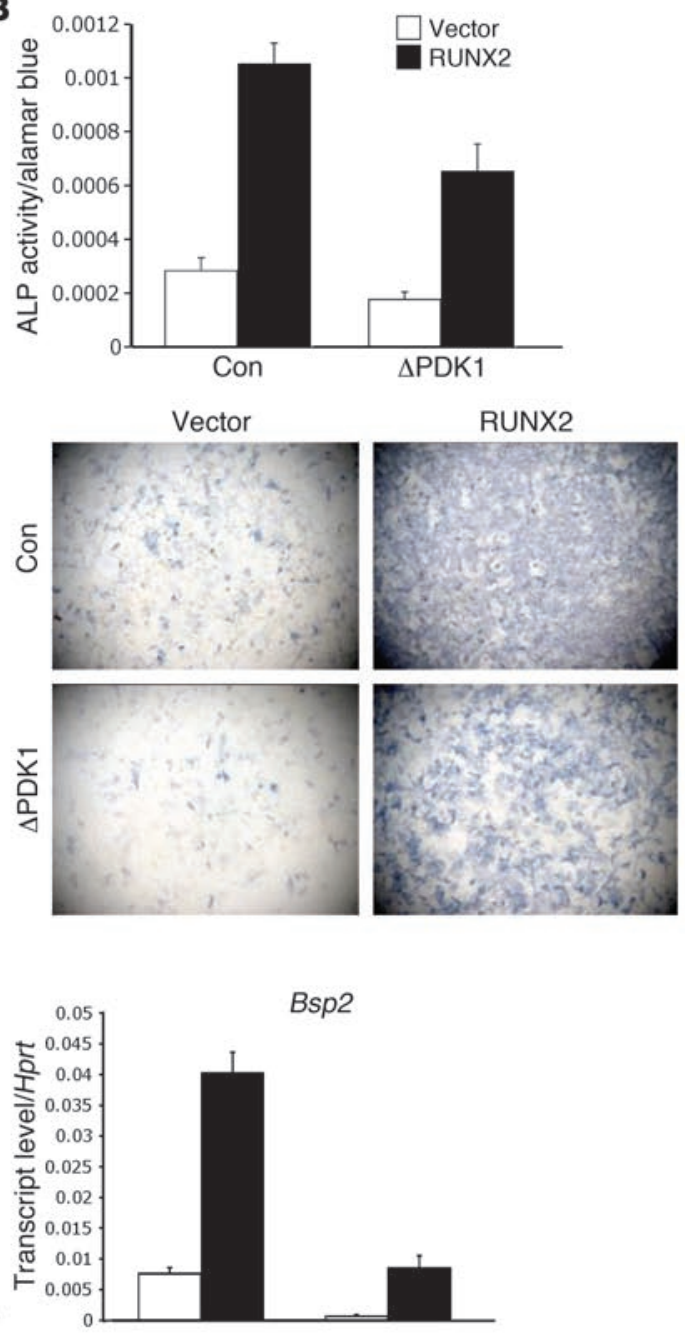

C


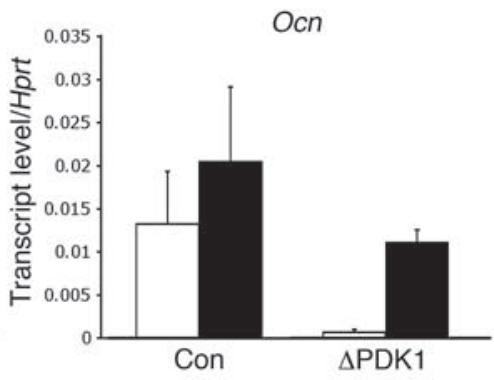

\section{Figure 7}

PDK1 functions upstream of RUNX2 in vitro. (A) $P d k 1^{f \mid l f l}$ CalvObs infected by control or Cre-expressing lentivirus were transfected with OSE2-Luc or OSE1-Luc along with Renilla luciferase gene. Luciferase activity was analyzed 6 days after culture in differentiation medium. Results were normalized to a Renilla control. (B and C) $P d k 1^{f \mid f l}$ CalvObs were infected with either control or Cre-expressing lentivirus, together with Myc-RUNX2-expressing lentivirus, and (B) analyzed by colorimetric assay (top) and Fast Blue staining (bottom) for ALP activity. Original magnification, $\times 100$. (C) Alternatively, RNA transcript levels of the indicated genes were analyzed by quantitative PCR.
(51-53). Given the evidence that BMP2 expression is downstream of the PDK1 pathway in osteoblasts, we tested whether the phenotype of $P d k 1^{o s x}$ mice can be rescued by treatment with rhBMP2/7. We used rhBMP2/7 instead of rhBMP2, as the rhBMP2/7 heterodimer is more potent than the rhBMP2 monomer (54). It has been reported that iodinated BMP7 injected into pregnant mice was able to pass across the placenta and successfully localized in developing fetal organs (55). Thus, we treated pregnant mice with either rhBMP2/7 (1 $\mu \mathrm{g}$ per mouse per day) or $0.1 \%$ BSA/PBS daily from E8.5 to E18.5 and sacrificed neonates at birth for analysis of skeletal phenotypes. Remarkably, treatment with rhBMP2/7 partially reversed the entire spectrum of skeletal anomalies seen in $P d k 1^{\text {osx }}$ pups, including rescue of calvarial hypomineralization, clavicular hypoplasia, and spontaneous fracture of the femur (Figure 11A). Furthermore, the defects in differentiation and marker gene expression seen in PDK1-deficient osteoblasts were partially reversed by rhBMP2/7 in vitro (Figure 11B). Thus, rhBMP2/7 treatment partially reverses the PDK1 phenotype both in vivo and in vitro, demonstrating that the PDK1 pathway is a physiological regulator of BMP2 expression.

Mutations in the Cbp gene cause RTS and $\mathrm{Cbp}^{+/-}$mice to recapitulate many of the skeletal features of RTS $(1,8,56,57)$. The finding that rhBMP2/7 can partially reverse the phenotype of $P d k 1^{o s x}$ mice suggests that a similar approach might be used to prevent the skeletal defects seen in the murine model of RTS, $\mathrm{Cbp}^{+/-}$mice. Similar to the strategy used above, pregnant mice 
A
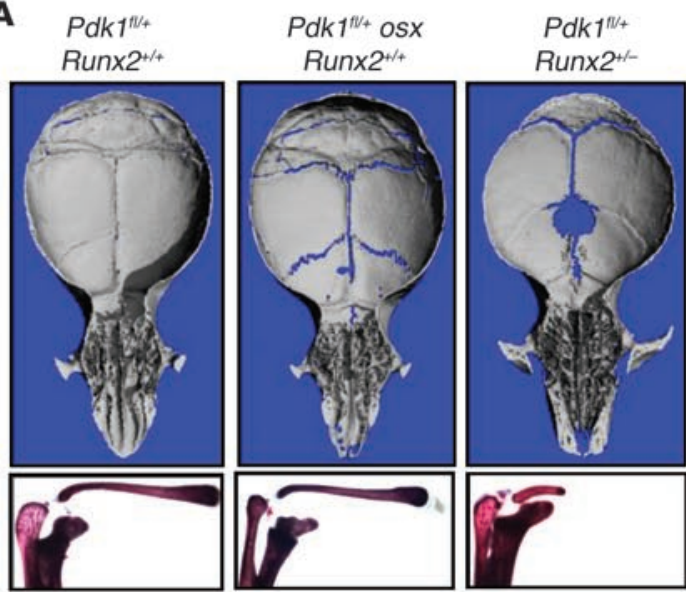

Pdk1 $1^{\text {H/ }}$ osx Runx2

B
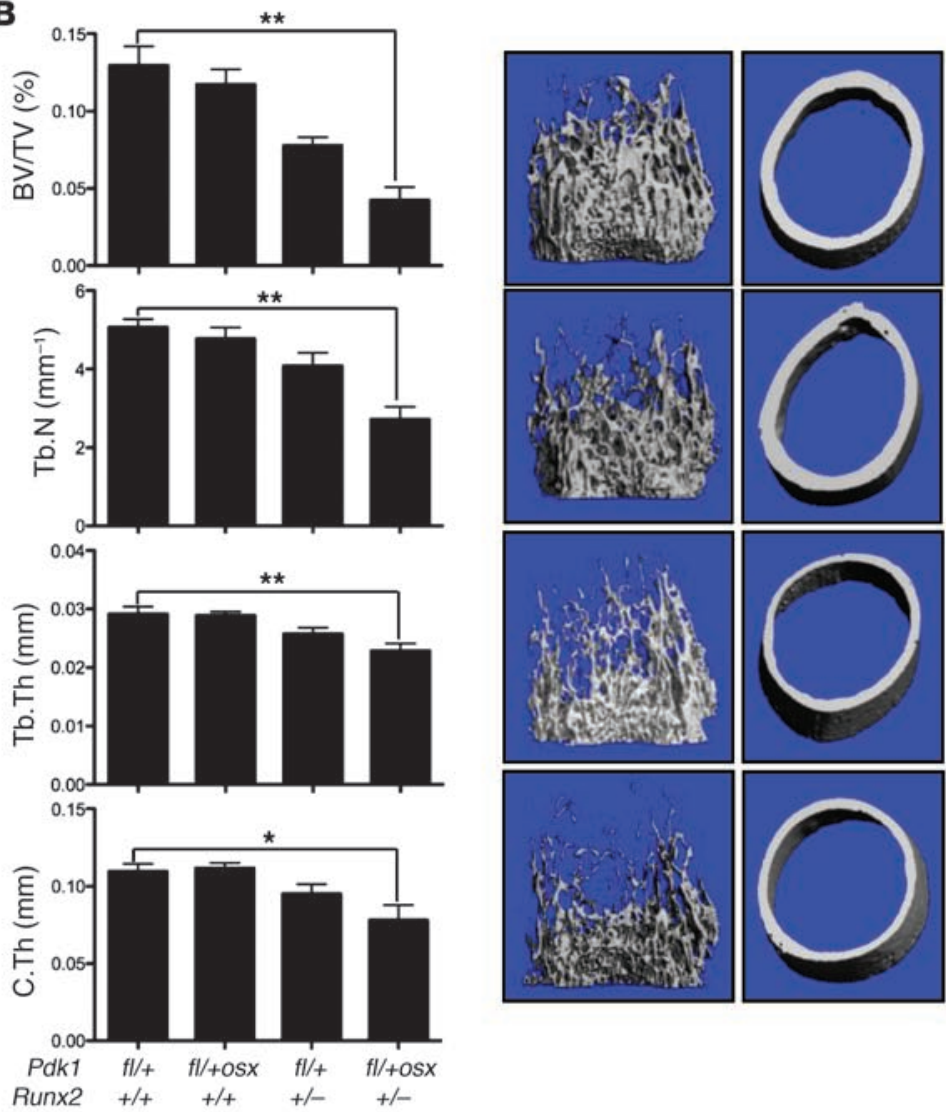

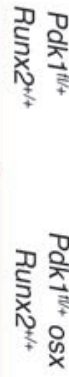
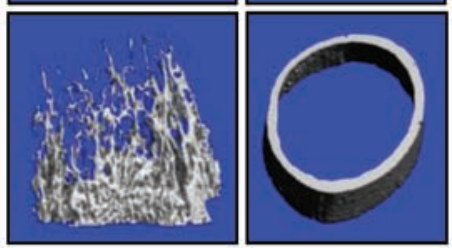

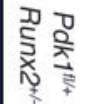

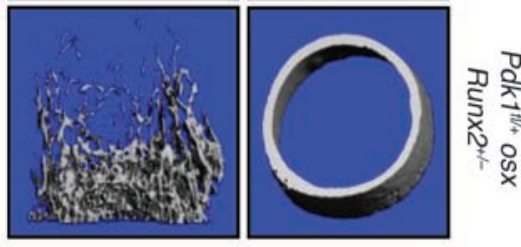

C

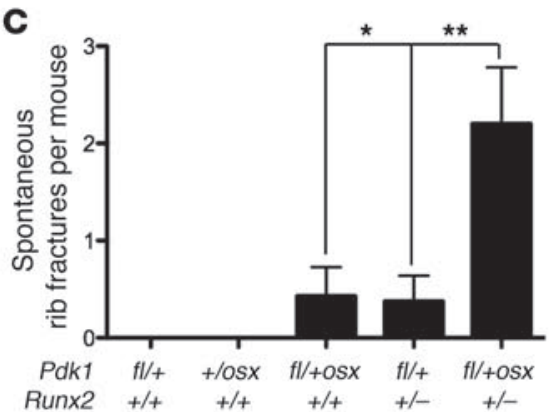

\section{Figure 8}

PDK1 regulates RUNX2 in vivo. (A) $\mu C T$ analysis of the calvaria (top) and Alizarin Red/Alcian Blue-stained skeletal preps of clavicles (bottom) from 4-week-old $P d k 1^{f l /+}, P d k 1^{f l+}$ osx, Run $x 2^{+/-}$, and Pdk $1^{f / /+}$ os $x$; Run $x 2^{+/-}$ mice. (B) $\mu \mathrm{CT}$ analysis of the femurs from 4-week-old $P d k 1^{1 / /+}, P d k 1^{f / /+}$ Osx, Runx $2^{+/-}$, and Pdk $1^{H /+}$ osx; Run $x 2^{+/-}$ mice. 3D reconstructions of trabecular bone (left) and midshaft cortical bone (right) are displayed. ${ }^{*} P<0.05$, Student's $t$ test. ${ }^{* \star} P<0.005$. (C) Spontaneous rib fractures of 4-week-old Pdk $1^{f l++}, P d k 1^{f l+}+o s x$, Runx $2^{+/-}$, and $P d k 1^{f / /}+0 s x$;Run $x 2^{+/-}$mice. ${ }^{*} P<0.05$, Student's $t$ test. ${ }^{* \star} P<0.005$.

were treated daily with rhBMP2/7 at gestational ages E8.5 through E17.5 and sacrificed at E18.5 for analysis of skeletal phenotypes. Treatment with rhBMP2/7 successfully ameliorated the skeletal anomalies seen in $\mathrm{Cbp}^{+/-}$embryos at a high frequency, including calvarial hypomineralization and clavicular hypoplasia (Figure 12A). Rescue of the CBP phenotype with rhBMP2/7 treatment in vivo demonstrates that the CREB/CBP pathway is a key biological regulator of BMP2 expression and provides proof of principle that in utero BMP2/7 administration may be a treatment strategy for heritable skeletal dysplasia syndromes.

\section{Discussion}

Our study defines a PDK1-dependent pathway in osteoblasts that acts to connect IGF-1 and insulin signaling to transcriptional activity of the CREB/CBP complex via AKT, thereby upregulating RUNX2 activity and BMP2 expression. Comparison of $P d k 1^{o s x}$ and $P d k 1^{d m 1}$ phenotypes indicates that this pathway likely functions both in osteoblast precursors and in later stage osteoblasts. Accordingly, treatment of $P d k 1$ conditional knockout or $\mathrm{Cbp}^{+/-}$embryos in utero with rhBMP2/7 partially reverses their skeletal defects, demonstrating both that BMP2 is a physiological target of a PDK1AKT-CREB/CBP signaling axis and that growth factor supplementation is able to correct skeletal dysplasia phenotypes in utero. However, despite the remarkable functional importance of BMPs, the CBP/CREB complex downstream of PDK1 almost certainly has additional targets, likely including many of the osteoblast marker genes affected by the absence of PDK1.

PDK1 is a potential causative gene of RTS. The function of IGF-1 and insulin in regulating adult bone mass may be mediated by the PDK1-AKT-CREB/ CBP pathway, offering new targets for the treatment of osteoporosis. Furthermore, the finding that $\mathrm{CBP}$ acts as an adjunct to CREB suggests that RTS (5) reflects the skeletal effects of disrupting the PDK1-AKT-CREB/CBP signaling axis. Whether impairment of this signaling axis in other tissues would also phenocopy the nonskeletal features of RTS is unknown. 


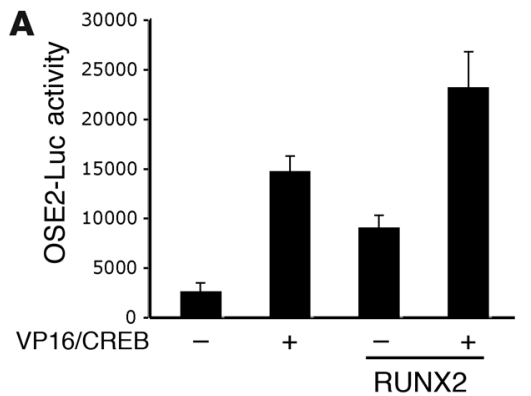

B

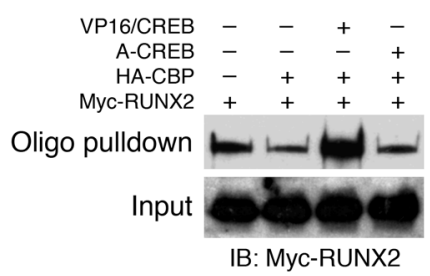

C

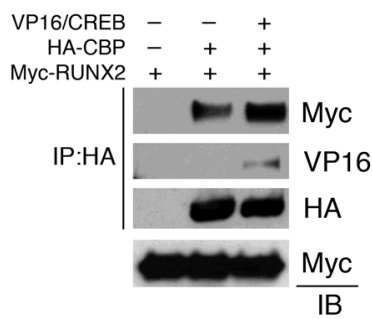

D

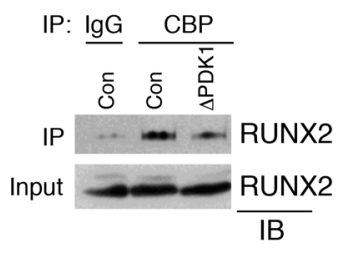

Figure 9

PDK1 regulates RUNX2 activity and BMP2 expression via the CREB/CBP complex. (A) C3H10T1/2 cells were transfected with the indicated constructs. Luciferase activity was measured and normalized to Renilla control. (B) A DNA pull-down assay with a biotinylated oligo containing the RUNX2-binding site (OSE2). HEK293 cells were transfected with Myc-RUNX2 in combination with HA-CBP, VP16/CREB, or A-CREB. Nuclear proteins were immunoprecipitated by a biotinylated OSE2 oligo and immunoblotted with anti-Myc antibody. The amount of Myc-RUNX2 protein was measured as an input control. (C) RUNX2 interaction with CBP is increased by the constitutively active CREB. HEK293 cells were transfected with Myc-RUNX2 in combination with VP16/CREB and HA-CBP. Cells were lysed, immunoprecipitated with anti-HA-conjugated beads, and immunoblotted with the indicated antibodies. (D) Primary Pdk $1^{\mathrm{fl} / \mathrm{fl}}$ CalvObs were infected with either control or Cre-expressing lentivirus and cultured under differentiation conditions. Nuclear proteins were immunoprecipitated by either IgG or anti-CBP and immunoblotted with anti-RUNX2.

Approximately $40 \%$ and $3 \%$ of patients with RTS have been reported to carry heterozygous mutations in either the Cbp gene or the gene encoding $p 300$, a CBP paralog, respectively $(5,58)$. Intriguingly, the human PDK1 gene is also located in the same chromosomal $16 \mathrm{p} 13.3$ region associated with RTS, approximately $1.1 \mathrm{Mbp}$ apart from the $5^{\prime}$ end of the Cbp gene. Given that microdeletion at chromosome $16 \mathrm{p} 13.3$ is frequently observed in RTS (59), a search for null or hypomorphic mutations of the PDK1 gene in patients with RTS without an identifiable CBP lesion may be warranted.

$P D K 1$ is a key regulator downstream of IGF-1 and insulin signaling in osteoblasts. Although the role of insulin family members in regulating osteoblast activation has been a subject of much interest recently, how this signal is integrated to alter the osteoblast transcriptional program remains to be fully elucidated. Here, we demonstrate that PDK1 functions selectively to regulate signal transduction by insulin family members, including IGF-1 and insulin, in osteoblast biology. First, PDK1 acts downstream of IGF-1 to regulate a subset of AKT substrates, especially the phosphorylation of CREB at S133. Biochemical and genetic interaction studies confirm that this PDK1-AKT pathway acts through CREB phosphorylation to regulate osteoblast differentiation.

Notably, our findings contrast with previous in vitro studies showing that the PI3K/AKT pathway regulates osteoblast differentiation through upregulation of RUNX2 expression but not RUNX2 transcriptional activity (31-34). This discrepancy might result from artifacts of in vitro experiments using osteoblast cell lines and inhibitors. Alternatively, whereas a broad range of AKT substrates are affected by treatment with inhibitors or RNAiinduced knockdown in cell lines, PDK1 deletion blocks activation of AKT substrates in a selective manner. Therefore, this selective signaling impairment by PDK1 deletion might not affect RUNX2 expression but rather target RUNX2 activity in osteoblasts.

We also uncovered previously unappreciated roles for CREB in bone formation. As a RUNX2-independent pathway, CREB phosphorylation recruits its coactivator CBP to the promoter of BMP2 through its direct binding to CRE, thereby promoting BMP2 transactivation. Whereas prior studies have shown that BMP2 production is RUNX2 dependent (60), this is likely due to indirect effects of RUNX2 on the overall progression of osteoblast differentiation, as RUNX2 overexpression was unable to transactivate the $B M P 2$ gene (data not shown). Dysregulation of CREB/CBP activity may decrease endogenous BMP2 levels, thereby leading to skeletal anomalies, including delayed ossification during embryonic development and impaired fracture healing during adult life.

In insulin signaling pathways, PDK1 regulates RUNX2 transcriptional activity through downregulation of TWIST2, a negative regulator of RUNX2 (61). This role requires AKT activation and is independent of the CREB/CBP complex. Increased RUNX2 activity via TWIST2 downregulation upregulates osteocalcin expression and activity, leading to increased bone formation and glucose metabolism (37). Previous studies show that osteoblastspecific deletion of the insulin receptor results in reduced bone acquisition and metabolic anomalies due to decreased osteocalcin expression and activity $(18,37,62)$. Our findings build upon those observations by revealing that PDK1/AKT is the specific pathway by which insulin downregulates TWIST2. Thus, PDK1 is a major pathway for coupling insulin family signaling to multiple downstream pathways regulating osteoblast activity.

The use of in utero growth factor supplementation for skeletal dysplasias. As with nearly all skeletal dysplasias, considerable recent advances in the understanding of the genetics of RTS have failed to translate into therapeutic advances. This is despite demonstrations that embryonic developmental defects can in fact be corrected by directed supplementation of relevant growth factors (63). A major barrier to the implementation of such an approach is that a reliable method of fetal screening needs to be developed and implemented. Fetal ultrasound has an excellent capacity to visualize the degree of fetal skeletal development, and in utero screening for developmental skeletal dysplasias is already the de facto standard of care in most developed countries. With respect to skeletal dysplasias, this technology has yet to be coupled with therapeutic interventions due to the absence of a method to intervene, even if the particular disorder tracking within a pedigree is known. Here, we provide proof of principle that growth factor supplementation in utero can correct skeletal dysplasias. In particular, we demonstrate that rhBMP2/7 partly corrects the skeletal abnormalities seen in a mouse model of 

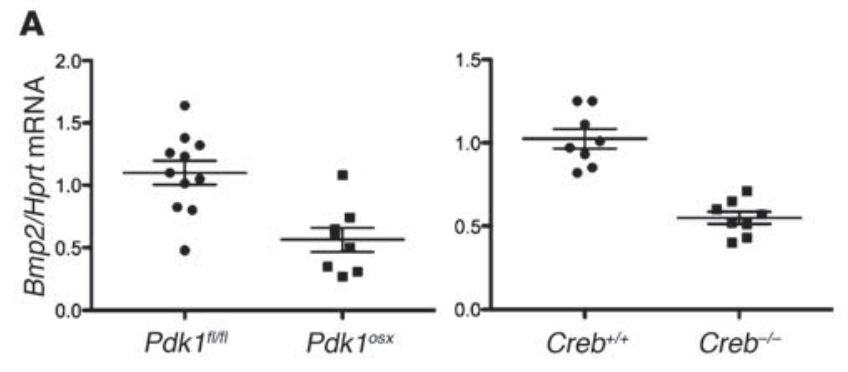

C

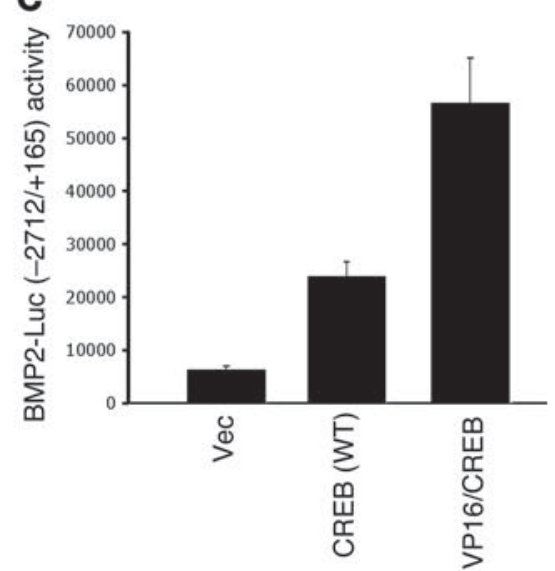

D
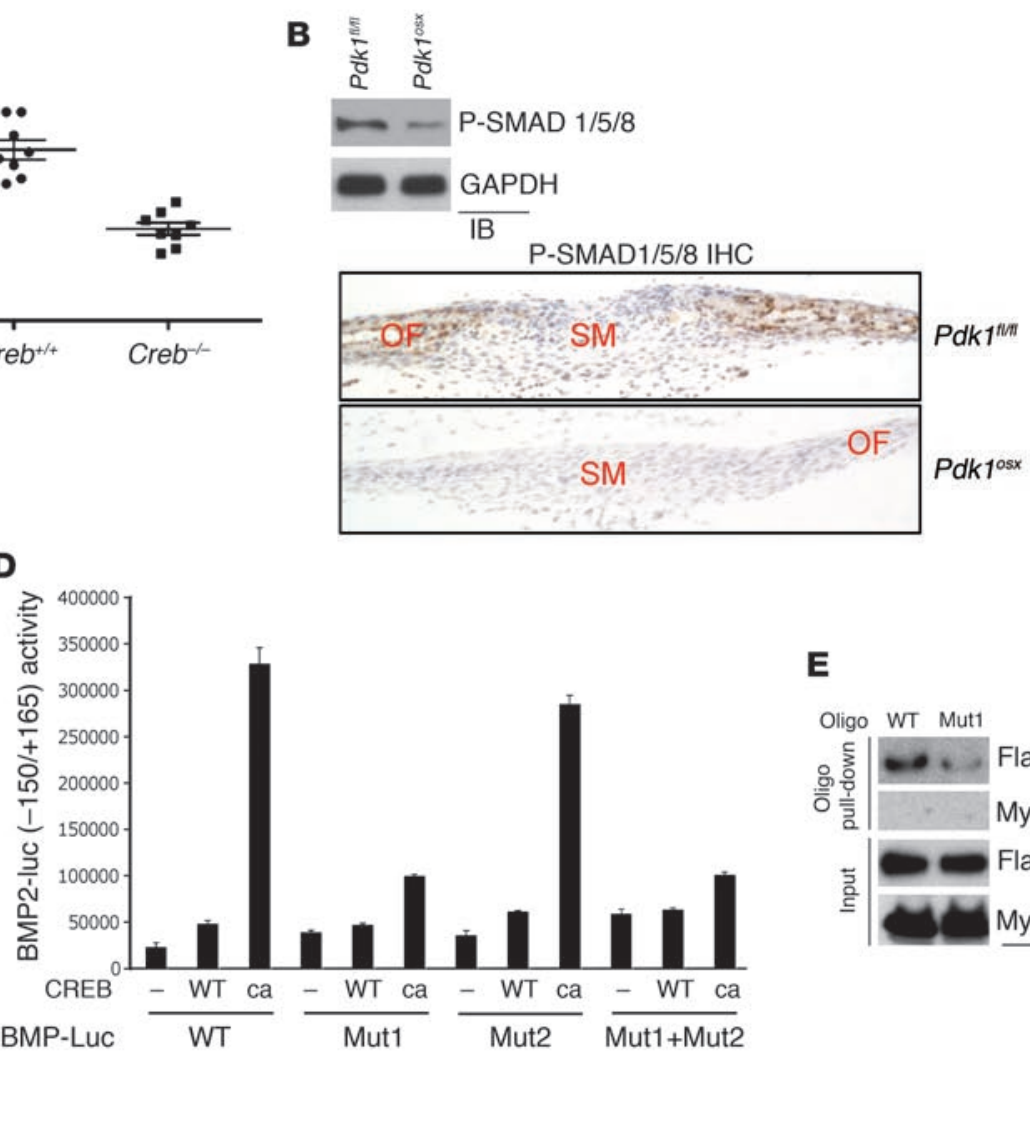

E

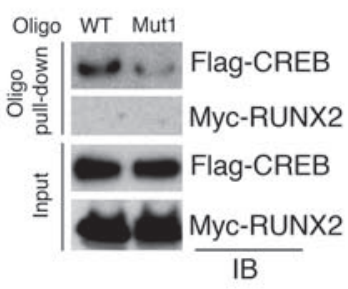

Figure 10

BMP2 is a target of the PDK1/CREB axis. (A) Total RNA was extracted from calvaria of P2 Pdk1 $1^{f l / f l}$ and Pdk $1^{\text {osx }}$ mice or E18.5 Crebl/t and $\mathrm{Creb}^{-/}$embryos. Transcript levels of $\mathrm{Bmp} 2$ were analyzed by quantitative PCR. Each dot represents the value from an independent embryo. The horizontal line represent mean \pm SD. (B) Primary CalvObs were isolated from $P d k 1^{f l l f l}$ and $P d k 1^{o s x}$ pups, lysed, and immunoblotted with anti-phospho-Smad1/5/8 antibody. Alternatively, IHC shows phosphorylation levels of SMAD1/5/8 in a coronal section of the calvaria of P2 Pdk1 ${ }^{\mathrm{fllt} f}$ and Pdk10sx mice. Original magnification, $\times 40$. (C) C3H10T1/2 cells were transfected with the BMP2-Luc ( $-2712 /+165 \mathrm{bp})$ and Renilla luciferase genes, along with vector, Flag-CREB (WT), or VP16/CREB. Results are expressed as relative luciferase activity normalized to Renilla control. (D) C3H10T1/2 cells were transfected with either WT or mutant BMP2-Luc $(-150 /+165$ bp) containing the CREB-binding site mutations (mut1 or mut2; mut1, -66/62 bp; mut2, +38/42 bp; WT, CGTCC; mutant, GGGCC) and Renilla luciferase genes along with Flag-CREB (WT) or VP16/CREB (ca). Results are expressed as relative luciferase activity normalized to Renilla control. (E) HEK293 cells were transfected with either Flag-CREB or Myc-RUNX2, and nuclear proteins were immunoprecipitated by biotinylated oligos containing the WT or mutant CREB-binding site.

RTS. Thus, understanding the targets downstream of CBP enables directed growth factor replacement, an approach which may be generalizable to developmental disorders outside of RTS. Additionally, these insights may also translate into biomarker discovery for RTS, allowing improved diagnostics beyond the current methods of $C B P$ sequencing or FISH for 16p13 deletions.

\section{Methods}

Cells, plasmids, and antibodies. hMSCs were cultured under osteoblast differentiation conditions, as instructed by the user's manuals (Lonza). Primary CalvObs and the C3H10T1/2 cell line (mouse mesenchymal fibroblast-like cell line) were cultured in $\alpha$-MEM medium (Cellgro) containing $10 \%$ FBS, $2 \mathrm{mM}$ L-glutamine, $1 \%$ penicillin/streptomycin, $1 \%$ HEPES, and $1 \%$ nonessential amino acids and differentiated with ascorbic acid and $\beta$-glycerophosphate. HEK293 cells (human kidney embryonic cells) and HEK293 FT cells were purchased from ATCC and Invitrogen, respectively. Myc-PDK1 (WT, K111A, and L155E), VP16/CREB, A-CREB, and mouse BMP2-Luc $(-150 /+165$ bp and $-2712 /+165$ bp) cDNAs were gifts from Dan R. Littman
(New York University, New York, New York, USA), Angel Barco (Instituto de Neurociencias de Alicante, Alicante, Spain), Charles Vinson (National Cancer Institute, Bethesda, Maryland, USA), and Stephen E. Harris (University of Texas Health Science Center at San Antonio), respectively. Flag-CREB (WT) cDNA was a gift from Marc Montminy (Addgene plasmid no. 22968). Cre recombinase, Myc-PDK1 (WT and K111A), and Myc-RUNX2 cDNAs were PCR amplified and cloned into pHASE/PGK-PURO lentiviral vector. The following antibodies were used: anti-PDK1, anti-phospho-AKT (T308 and S473), anti-phospho-GSK-3 $\beta$ (S9), anti- $\beta$-catenin, anti-phospho-AKT substrates (RXXS/T*), anti-phospho-S6 (S235/236), anti-phospho-4EBP1 (T37/T46), anti-phospho-CREB (S133), anti-phospho-SMAD1/5/8, anti-phospho-ERK1/2, anti-phospho-JNK1/2, and anti-phospho-p38 (all from Cell Signaling Technology); anti-HSP90, anti-HA-conjugated agarose, anti-c-Myc-conjugated HRP, and anti-HA-conjugated HRP (all from Santa Cruz Biotechnology Inc.); anti-Flag (M2) and anti-VP16 (both from Sigma-Aldrich); anti-CBP, anti-Tie2, anti-CD45, anti-Ter119, antiCD105, and anti-Thy1.2 (all from Biolegend); anti-RUNX2 (Calbiochem); and anti-GAPDH (Affinity Bioreagents). As indicated, cells were treated 
A
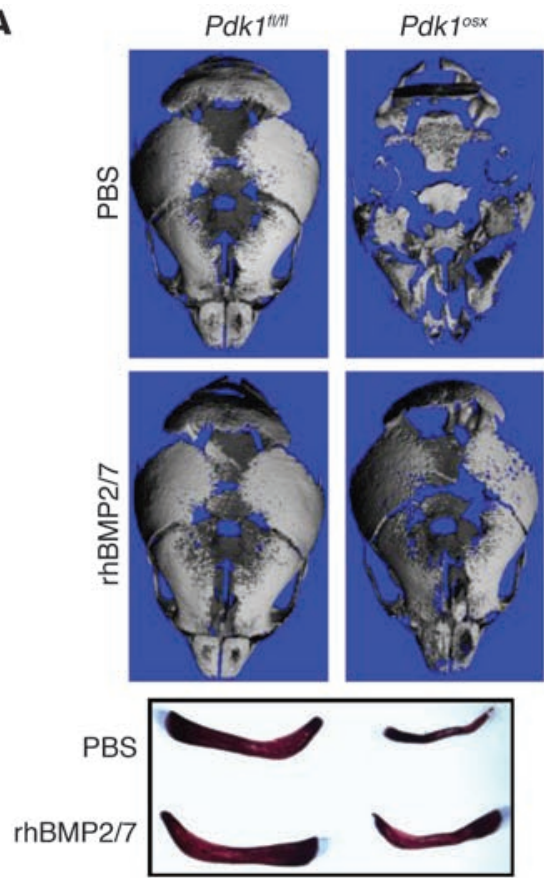

B

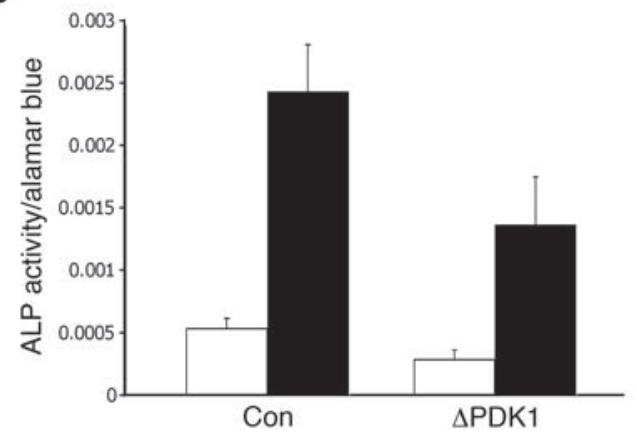

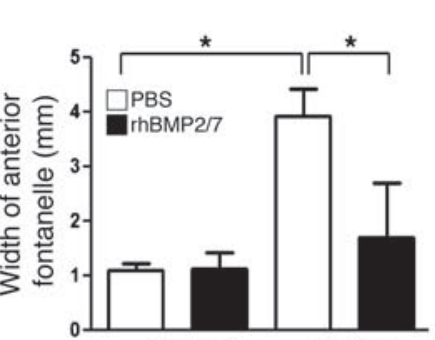

Pdk1 $1^{\text {fMI }}$

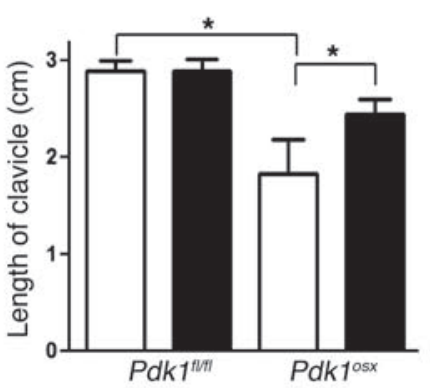

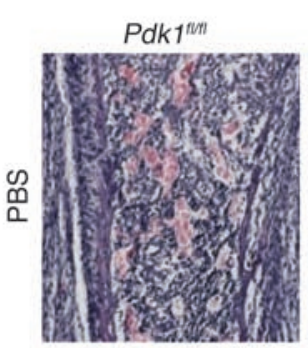

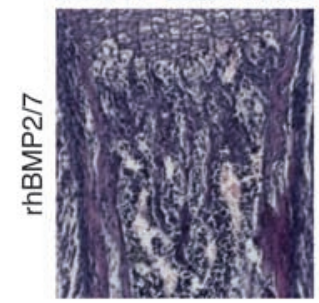

Pdk1osx
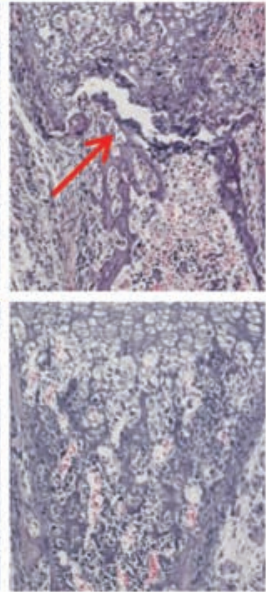

PBS

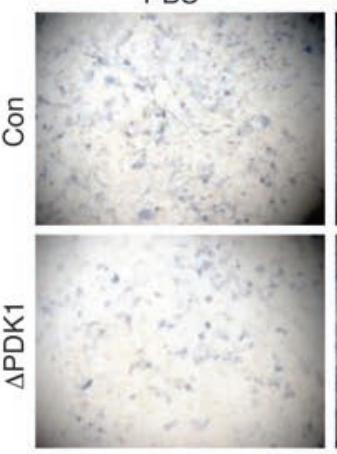

rhBMP2/7

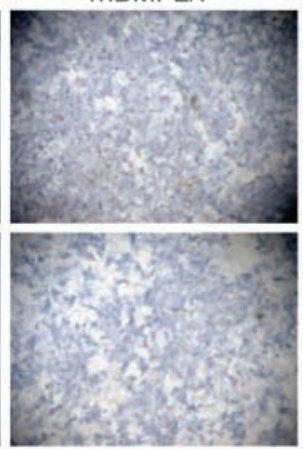

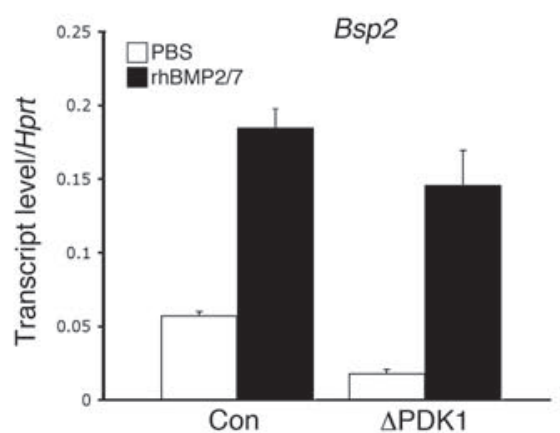
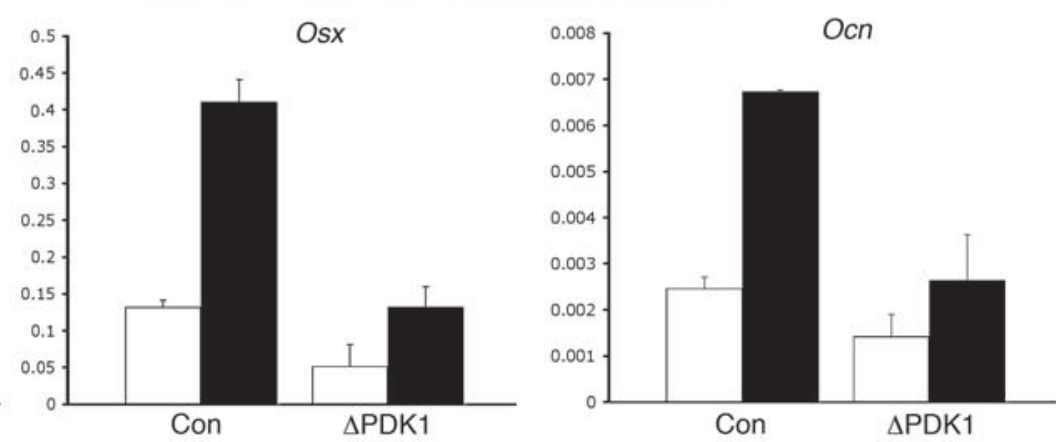

\section{Figure 11}

rhBMP2/7 treatment reverses skeletal anomalies in Pdk10sx embryos and osteoblasts. (A) Rescue of skeletal anomalies in P1 Pdk1osx neonates by rhBMP2/7. Ossification of P1 Pdk10sx skulls treated with either $0.1 \%$ BSA/PBS or rhBMP2/7 was analyzed by $\mu \mathrm{CT}$ (top). Alizarin Red/Alcian Blue-stained skeletal preps of the clavicle (middle) and hematoxylin and eosin-stained P1 Pdk $1^{\text {fllfl }}$ and Pdk10sx femurs (bottom) are shown. The arrow indicates a spontaneous fracture. Original magnification, $\times 100$. ${ }^{\star} P<0.01$, Student's $t$ test; $P<0.02$ (for clavicle measurements) and $P<0.0001$ (for fontanelle measurements), 1-way ANOVA. (B) Rescue of impaired differentiation in PDK1-deficient CalvObs by rhBMP2/7. Pdk $1^{f / l f t}$ CalvObs were infected with control or Cre-expressing lentivirus and cultured for 6 days under in the presence of $0.1 \%$ BSA/PBS or rhBMP2/7 (100 ng/ml). ALP activity and RNA transcript levels of the indicated genes were analyzed. Original magnification, $\times 40($ A, right $) ; \times 100(B)$. 
A
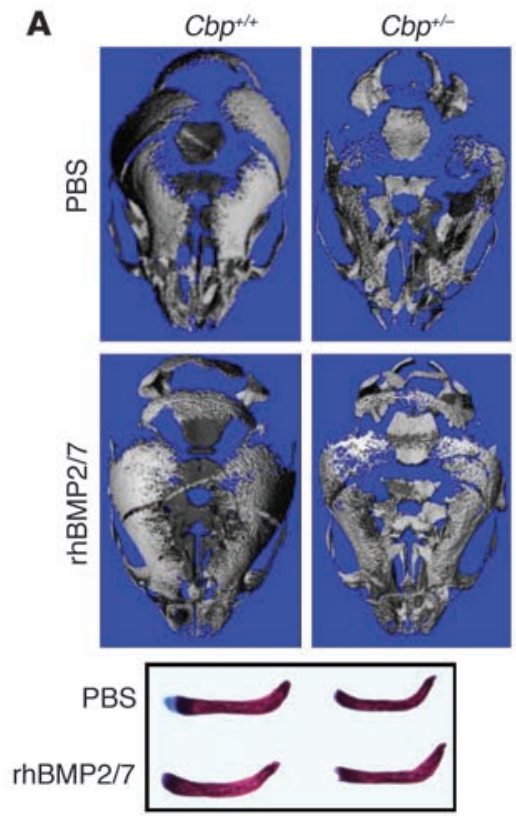

B


Figure 12

rhBMP2/7 treatment reverses skeletal anomalies in $\mathrm{Cbp}^{+/-}$embryos. (A) Rescue of skeletal anomalies in E18.5 $\mathrm{Cbp}^{+/-}$embryos by rhBMP2/7. Ossification of $\mathrm{E} 18.5 \mathrm{Cbp}^{+/}$- skulls treated with either $0.1 \% \mathrm{BSA} / \mathrm{PBS}$ or rhBMP2/7 was analyzed by $\mu \mathrm{CT}$, and the $3 \mathrm{D}$ reconstruction is displayed (top). Alizarin Red/Alcian Blue-stained skeletal preps demonstrate a rescue of clavicular hypoplasia in $\mathrm{Cbp}^{+/}$embryos treated with rhBMP2/7 (bottom). $P<0.0001,1$ way ANOVA; ${ }^{*} P<0.01$, Student's $t$ test. (B) Schema depicting the PDK1 pathway in osteoblasts. Black arrows indicate the IGF-1 signaling pathway; red arrows and lines indicate the insulin-signaling pathway.

with rhBMP2/7, human IGF-1, human FGF-2, and murine TGF- $\beta$ (R\&D Systems); insulin, forskolin, and isoproterenol (Sigma-Aldrich); and inhibitors for PI3K, PDK1, and AKT (Calbiochem).

Breeding of mouse strains. Pdk $1^{f l / f l}$ mice were intercrossed with the Osx-cre deleter strain (a gift from Andrew McMahon, Harvard University, Boston, Massachusetts, USA) to generate $P d k 1^{o s x}$ mice (25). Run $x 2^{+/-}$mice were gifts from Bjorn Olsen (64), and $\mathrm{Creb}^{+/-}$mice and the Dermo1-cre deleter strain were purchased from The Jackson Laboratory. All mice analyzed, including Pdk1 $1^{o s x}, \mathrm{Run}^{2+/-}, \mathrm{Creb}^{+/-}$, and $\mathrm{Cbp}^{+/-}$mice, were maintained on the C57BL/6 background.

Skeletal preparation and $\mu C T$ analysis. For skeletal preparation, skeletons were prepared for gross analysis using the method of McLeod (65). Briefly, mice were skinned, eviscerated, and fixed in $95 \%$ ethanol. Then, skeletons were stained by Alizarin Red S/Alcian Blue and sequentially cleared in $1 \%$ potassium hydroxide.

For $\mu \mathrm{CT}$ analysis, a Scanco Medical $\mu \mathrm{CT} 35$ system with an isotropic voxel size of $7 \mu \mathrm{m}$ was used to image the distal femur. Scans were conducted in $70 \%$ ethanol and used an X-ray tube potential of $55 \mathrm{kVp}$, an X-ray intensity of $0.145 \mathrm{~mA}$, and an integration time of $600 \mathrm{~ms}$. From the scans of the distal femur, a region beginning 0.28 millimeters proximal to the growth plate and extending 2.1 millimeters proximally was selected for trabecular bone analysis. A second region 0.6 millimeters in length and centered at the midpoint of the femur was used to calculate diaphyseal parameters. A Gaussian filter, with a support of 0.8 and sigma of 1 , was applied to the femur images. In order to distinguish cortical from trabecular bone, a semiautomated contouring approach was used. A contour was drawn by hand a few voxels from the endocortical surface every 20 slices. Contours on slices in this range were interpolated by the software. The region of interest was thresholded using a global threshold that set the bone/marrow cutoff at $438.5 \mathrm{mgHA} /$ $\mathrm{cm}^{3}$ for trabecular bone and $589.4 \mathrm{mgHA} / \mathrm{cm}^{3}$ for cortical bone. Unbiased,
3D microstructural properties of trabecular bone, including bone volume fraction, trabecular thickness, trabecular number, and trabecular separation, were then calculated for the trabecular region of the metaphysis of the distal femur as well as midshaft bone volume fraction and cortical thickness for the diaphysis, using methods based on distance transformation of the binarized images (66). Scans of the skulls were performed using isotropic voxel sizes of $12 \mu \mathrm{m}$ and $20 \mu \mathrm{m}$. A Gaussian filter, with a support of 0.8 and sigma of 1 , was applied to the skull images. A contour was created that encompassed the entire skull, and the volume of interest was thresholded using a global threshold that set the bone/air cutoff at $136.8 \mathrm{mgHA} / \mathrm{cm}^{3}$. All images presented are representative of the respective genotypes.

Isolation of fetal osteoprogenitor cells. Fetal osteoprogenitor cells were isolated from E17.5 $P d k 1^{f / f l}, P d k 1^{o s x}, P d k 1^{d m 1}$, or $P d k 1^{f l++} d m 1$ embryos as previously described (30). Briefly, both hind and fore limbs from E17.5 embryos were digested with type I collagenase (Sigma-Aldrich) and dispase II (Roche) for 40 minutes at $37^{\circ} \mathrm{C}$. The cells were stained with antibodies specific to Tie2, CD45, Ter119, Cd105, $\alpha_{v}$-integrin, and Thy 1.2 after lysing red blood cells. Data were acquired on LSRII and analyzed using Flowjo Flow Cytometry Analysis software.

Inducible PDK1 deletion and osteoblast differentiation analysis. Primary osteoblast precursors were isolated from calvaria of $\mathrm{P} 2 \mathrm{Pdk} 1^{f / / l}$ neonates by using the digestion solution containing type I collagenase (Sigma-Aldrich) and dispase II (Roche). For inducible deletion of the Pdk1 gene, $P d k 1^{f l / f l}$ osteoblast precursors were transduced with either EGFP-expressing lentivirus (control) or Cre-recombinase-expressing lentivirus (Cre) at the same MOI and cultured in osteoblast differentiation media containing ascorbic acid and $\beta$-glycerophosphate. Forty-eight hours after infection, the transduced cells were selected by puromycin.

For osteoblast differentiation assay, ALP activity, extracellular matrix mineralization, and osteoblast marker gene expression were analyzed as 
previously described (67). Briefly, for ALP staining, osteoblasts were fixed with $10 \%$ neutral buffered formalin and stained with a solution containing Fast Blue and Naphthol (Sigma-Aldrich). Alternatively, osteoblasts were incubated with Alamar Blue solution, washed, and incubated with a phosphatase substrate solution (Sigma-Aldrich), and ALP activity was measured by luminometer (Thermo Electron Co.). For Von Kossa staining of extracellular matrix mineralization, cells were fixed with $10 \%$ neutral buffered formalin and stained with a solution containing $2.5 \%$ silver nitrate (Sigma-Aldrich). For osteoblast marker gene expression, total mRNAs were purified from osteoblast cultures for use in quantitative RTPCR reactions that measure the expression level of several genes that are regulated during osteoblast differentiation.

Histology, in situ bybridization, and IHC. For histological analyses, paraffin sections of bones were produced from E16.5, E18.5, P1, and 4-weekold mice. Limb tissues were dissected and fixed in $4 \%$ paraformaldehyde in PBS. They were then decalcified by daily changes of $15 \%$ tetrasodium EDTA, until soft and pliable. Tissues were dehydrated by passage through an ethanol series, cleared twice in xylene, embedded in paraffin, and sectioned. For morphological analyses, tissue sections were stained with hematoxylin and eosin.

For in situ hybridization, DIG-labeled probes were prepared to detect osteocalcin and osteopontin mRNA expression using the DIG-labeling Kit (Roche), per the manufacturer's instructions, and the probes were hybridized with paraffin sections. DIG-labeled probe was then detected by immunostaining with anti-DIG-POD and streptadvidin-HRP (67).

For IHC, paraffin tissue sections were dewaxed, and endogenous peroxidase was quenched, as in preparation for in situ hybridization. Sections were blocked with $3 \%$ goat serum, $1 \%$ BSA, $0.1 \%$ Triton X-100 in PBS for 1 hour at room temperature and incubated with antibodies specific for PDK1, phospho-AKT (T308), phospho-GSK-3 $\beta$ (S9), phospho-S6, phospho-CREB (S133), and phospho-SMAD $1 / 5 / 8$ at $4^{\circ} \mathrm{C}$ overnight. Sections were then treated with TSA-biotin (Perkin Elmer) and streptavidin-HRP, as per manufacturer's instructions, and HRP visualized with 2,2'-diaminobenzidine tetrahydrochloride.

Luciferase reporter assay. Luciferase reporter assay was performed as described previously (67). Briefly, C3H10T1/2 cells were transfected using Effectene (Qiagen) with the indicated reporter genes and the Renilla luciferase gene (Promega), together with plasmids of VP16/CREB, Flag-CREB (WT), CBP, or RUNX2. Total DNA concentration in each experiment was maintained by adding the appropriate control vector to the DNA mixture. Forty-eight hours after transfection, cells were lysed, and luciferase activity was measured using the Dual-Luciferase Assay Kit (Promega). Alternatively, primary osteoblast precursors isolated from calvaria of $P d k 1^{f / f l}$ neonates were transduced with either EGFP- or Cre-recombinase-expressing lentivirus and cultured in the medium containing puromycin. Puromycin-resistant cells were transfected using Effectene (Qiagen) with various reporter genes and the Renilla luciferase gene. Six days after culture in differentiation medium, cells were lysed, and luciferase activity was measured using the Dual-Luciferase Assay Kit (Promega).

DNA binding assay. DNA binding assay was performed as described previously (68). Briefly, HEK293 cells were transfected with either FlagCREB or Myc-RUNX2 cDNA, and nuclear proteins were extracted from the cells by using nuclear and cytoplasmic extraction reagents (Thermo
Scientific). $150 \mu \mathrm{g}$ nuclear protein was incubated with biotinylated probe conjugated to streptavidin-agarose (Invitrogen) bound to protein containing the RUNX2-binding (OSE2-binding) site and WT or mutant CREBbinding site (CRE) for 1 hour at $4^{\circ} \mathrm{C}$ in binding buffer $(100 \mathrm{mM} \mathrm{NaCl}, 10$ mM Tris-HCl [pH 7.6], $0.1 \mathrm{mM}$ EDTA, 1 mM DTT, $5 \%$ glycerol, $1 \mathrm{mg} / \mathrm{ml}$ BSA, $20 \mu \mathrm{g} / \mathrm{ml}$ poly $\mathrm{dI} / \mathrm{dC}$ plus protease inhibitors). The coimmunoprecipitates were washed 3 times in binding buffer, resolved by SDS-PAGE, and immunoblotted for overexpressed Flag-CREB and Myc-RUNX2. The following primer sequences were used: OSE2-WT, GATCCGCTGCCATCACCAACCACAGCA; CRE-WT, CGCGGCCCAGCTAACGCAGAACGTCCGTCCCTCGCCCGGCGAG; and CRE-MUT, CGCGGCCCAGCTAACGCAGAAGCCCCGTCCCTCGCCCGGCGAG.

Treatment of animals with rhBMP2/7. For treatment of animals with rhBMP2/7, $10 \mu \mathrm{g}$ rhBMP2/7 (R\&D Systems) was dissolved in a total volume of $1 \mathrm{ml}$, and a dose of $1 \mu \mathrm{g}$ rhBMP2/7 per day was injected intraperitoneally into pregnant mice on E8.5 through E18.5. Injection of PBS was used as a control.

Statistics. Where appropriate, 1-way ANOVA was performed, and 2-tailed, unpaired Student's $t$ tests were used as a post-test for the select biologically relevant comparisons. Otherwise, unpaired Student's $t$ tests were used directly. A $P$ value equal to or less than 0.05 was considered significant. All data graphed are represented as mean $\pm \mathrm{SD}$. Quantification of repeats for all Western blots shown is provided in Supplemental Figure 11.

Study approval. All animals were maintained in accordance with the NIH Guide for the Care and Use of Laboratory Animals and were handled according to protocols approved by the Harvard University and University of Texas Health Science Center at San Antonio subcommittees on animal care (IACUC).

\section{Acknowledgments}

We would like to thank Sankar Ghosh and Sung-Gyoo Park (Columbia University, New York, New York, USA) for providing Pdk1 floxed mice; Dorien J.M. Peters, Rachel H. Giles, Michael L. Cunningham, Ann-Hwee Lee, Marc Wein, Henry Kronenberg, Dallas Jones, and Antonios Aliprantis for helpful discussions; and Chantel Lester, Heather De Rivera, Bella Hu, and Kirsten Sigrist for technical support. We also thank the many individuals who provided valuable reagents. This work was supported by NIH grant HD055601 (to L.H. Glimcher); J.-H. Shim was supported by an Arthritis Foundation postdoctoral fellowship. This work was also supported by National Creative Research Initiatives, a grant from the National Research Foundation of Korea funded by a Korean government grant (2011-0000425 to S.-K. Lee) and the Brain Korea 21 (BK21) Program.

Received for publication June 13, 2011, and accepted in revised form October 19, 2011.

Address correspondence to: Laurie H. Glimcher or Jae-Hyuck Shim, FXB Rm 205, 651 Huntington Ave., Boston, Massachusetts 02115, USA. Phone: 617.432.0622; Fax: 617.432.1223; E-mail: lglimche@hsph.harvard.edu (L.H. Glimcher), jshim@ hsph.harvard.edu (J.-H. Shim).

\footnotetext{
1. Rubinstein JH, Taybi H. Broad thumbs and toes and facial abnormalities. A possible mental retardation syndrome. Am J Dis Child. 1963;105:588-608.

2. Stevens CA. Patellar dislocation in Rubenstein-Taybi syndrome. Am JMed Genet. 1997;72(2):188-190.

3. Bloch-Zupan A, Stachtou J, Emmanouil D, Arveiler B, Griffiths D, Lacombe D. Oro-dental features as useful diagnostic tool in Rubinstein-Taybi syn-

drome. Am J Med Genet A. 2007;143(6):570-573. 4. Breuning $\mathrm{MH}$, et al. Rubinstein-Taybi syndrome caused by submicroscopic deletions within 16p13.3. Am J Hum Genet. 1993;52(2):249-254.

5. Petrij F, et al. Rubinstein-Taybi syndrome caused by mutations in the transcriptional co-activator CBP. Nature. 1995;376(6538):348-351.

6. Wang J, et al. CBP histone acetyltransferase activity
}

regulates embryonic neural differentiation in the normal and Rubinstein-Taybi syndrome brain. Dev Cell. 2010;18(1):114-125.

7. Alarcon JM, et al. Chromatin acetylation, memory, and LTP are impaired in $\mathrm{CBP}^{+/-}$mice: a model for the cognitive deficit in Rubinstein-Taybi syndrome and its amelioration. Neuron. 2004;42(6):947-959.

8. Tanaka Y, Naruse I, Maekawa T, Masuya H, Shi- 
roishi T, Ishii S. Abnormal skeletal patterning in embryos lacking a single Cbp allele: a partial similarity with Rubinstein-Taybi syndrome. Proc Natl Acad Sci U S A. 1997;94(19):10215-10220.

9. Oike Y, et al. Truncated CBP protein leads to classical Rubinstein-Taybi syndrome phenotypes in mice: implications for a dominant-negative mechanism. Hum Mol Genet. 1999;8(3):387-396.

10. Chrivia JC, Kwok RP, Lamb N, Hagiwara M, Montminy MR, Goodman RH. Phosphorylated CREB binds specifically to the nuclear protein CBP. Nature. 1993;365(6449):855-859.

11. Du K, Montminy M. CREB is a regulatory target for the protein kinase Akt/PKB. J Biol Chem. 1998;273(49):32377-32379.

12. Nakajima $T$, et al. The signal-dependent coactivator CBP is a nuclear target for pp90RSK. Cell. 1996;86(3):465-474.

13. Perkins ND, Felzien LK, Betts JC, Leung K, Beach DH, Nabel GJ. Regulation of NF-kappaB by cyclindependent kinases associated with the p300 coactivator. Science. 1997;275(5299):523-527.

14. Langlois JA, et al. Association between insulin-like growth factor I and bone mineral density in older women and men: the Framingham Heart Study. J Clin Endocrinol Metab. 1998;83(12):4257-4262.

15. Turner $\mathrm{CH}$, et al. Genetic regulation of cortical and trabecular bone strength and microstructure in inbred strains of mice. J Bone Miner Res. 2000; 15(6):1126-1131

16. Kawai M, Rosen CJ. Insulin-like growth factor-I and bone: lessons from mice and men. Pediatr Nephrol. 2009;24(7):1277-1285.

17. Ferron $\mathrm{M}$, et al. Insulin signaling in osteoblasts integrates bone remodeling and energy metabolism. Cell. 2010;142(2):296-308.

18. Fulzele $\mathrm{K}$, et al. Insulin receptor signaling in osteoblasts regulates postnatal bone acquisition and body composition. Cell. 2010;142(2):309-319.

19. Ogata $\mathrm{N}$, et al. Insulin receptor substrate- 1 in osteoblast is indispensable for maintaining bone turnover. J Clin Invest. 2000;105(7):935-943.

20. Akune T, et al. Insulin receptor substrate- 2 maintains predominance of anabolic function over catabolic function of osteoblasts. J Cell Biol. 2002; 159(1):147-156.

21. Liu JP, Baker J, Perkins AS, Robertson EJ, Efstratiadis A. Mice carrying null mutations of the genes encoding insulin-like growth factor I (Igf-1) and type 1 IGF receptor (Igf1r). Cell. 1993;75(1):59-72.

22. Peng XD, et al. Dwarfism, impaired skin development, skeletal muscle atrophy, delayed bone development, and impeded adipogenesis in mice lacking Akt1 and Akt2. Genes Dev. 2003;17(11):1352-1365.

23. Lawlor MA, et al. Essential role of PDK1 in regulating cell size and development in mice. EMBO J. 2002;21(14):3728-3738.

24. Inoue $\mathrm{H}$, et al. Role of hepatic STAT3 in braininsulin action on hepatic glucose production. Cell Metab. 2006;3(4):267-275.

25. Rodda SJ, McMahon AP. Distinct roles for Hedgehog and canonical Wnt signaling in specification, differentiation and maintenance of osteoblast progenitors. Development. 2006;133(16):3231-3244.

26. Bartsch O, et al. Two patients with EP300 mutations and facial dysmorphism different from the classic Rubinstein-Taybi syndrome. Am J Med Genet A. 2010;152A(1):181-184.

27. Mundlos S. Cleidocranial dysplasia: clinical and molecular genetics. J Med Genet. 1999;36(3):177-182.

28. Hennekam RC, Stevens CA, Van de Kamp JJ. Etiology and recurrence risk in Rubinstein-Taybi syn- drome. Am J Med Genet Suppl. 1990;6:56-64.

29. Li L, Cserjesi P, Olson EN. Dermo-1: a novel twistrelated bHLH protein expressed in the developing dermis. Dev Biol. 1995;172(1):280-292.

30. Chan CK, et al. Endochondral ossification is required for haematopoietic stem-cell niche formation. Nature. 2009;457(7228):490-494.

31. Fujita T, et al. Runx2 induces osteoblast and chondrocyte differentiation and enhances their migration by coupling with PI3K-Akt signaling. J Cell Biol. 2004;166(1):85-95.

32. Kawamura N, et al. Akt1 in osteoblasts and osteoclasts controls bone remodeling. PLoS One. 2007; 2(10):e1058.

33. Mukherjee A, Rotwein P. Akt promotes BMP2mediated osteoblast differentiation and bone development. J Cell Sci. 2009;122(pt 5):716-726.

34. Mukherjee A, Wilson EM, Rotwein P. Selective signaling by Akt 2 promotes bone morphogenetic protein 2-mediated osteoblast differentiation. $\mathrm{Mol}$ Cell Biol. 2010;30(4):1018-1027.

35. Pearce LR, Komander D, Alessi DR. The nuts and bolts of AGC protein kinases. Nat Rev Mol Cell Biol. 2010;11(1):9-22.

36. Williams MR, et al. The role of 3-phosphoinositide-dependent protein kinase 1 in activating AGC kinases defined in embryonic stem cells. Curr Biol. 2000;10(8):439-448.

37. Fulzele K, et al. Insulin receptor signaling in osteoblasts regulates postnatal bone acquisition and body composition. Cell. 2010;142(2):309-319.

38. Li XY, Zhan XR, Liu XM, Wang XC. CREB is a regulatory target for the protein kinase Akt/PKB in the differentiation of pancreatic ductal cells into islet beta-cells mediated by hepatocyte growth factor. Biochem Biophys Res Commun. 2011;404(2):711-716.

39. Kato S, Ding J, Du K. Differential activation of CREB by Akt 1 and Akt2. Biochem Biophys Res Com mun. 2007;354(4):1061-1066

40. Cross DA, Alessi DR, Cohen P, Andjelkovich M, Hemmings BA. Inhibition of glycogen synthase kinase- 3 by insulin mediated by protein kinase B. Nature. 1995;378(6559):785-789.

41. Ruvinsky I, et al. Ribosomal protein S6 phosphorylation is a determinant of cell size and glucose homeostasis. Genes Dev. 2005;19(18):2199-2211.

42. Rudolph D, Tafuri A, Gass P, Hammerling GJ, Arnold B, Schutz G. Impaired fetal T cell development and perinatal lethality in mice lacking the cAMP response element binding protein. Proc Nat Acad Sci U S A. 1998;95(8):4481-4486.

43. Bleckmann SC, Blendy JA, Rudolph D, Monaghan AP, Schmid W, Schutz G. Activating transcription factor 1 and CREB are important for cell survival during early mouse development. Mol Cell Biol. 2002;22(6):1919-1925

44. Golan I, et al. Atypical expression of cleidocranial dysplasia: clinical and molecular-genetic analysis. Orthod Craniofac Res. 2002;5(4):243-249.

45. Barco A, et al. Gene expression profiling of facilitated L-LTP in VP16-CREB mice reveals that BDNF is critical for the maintenance of LTP and its synaptic capture. Neuron. 2005;48(1):123-137.

46. Ahn S, Olive M, Aggarwal S, Krylov D, Ginty DD, Vinson C. A dominant-negative inhibitor of CREB reveals that it is a general mediator of stimulusdependent transcription of c-fos. Mol Cell Biol. 1998; 18(2):967-977

47. Tsuji $\mathrm{K}$, et al. BMP2 activity, although dispensable for bone formation, is required for the initiation of fracture healing. Nat Genet. 2006;38(12):1424-1429. 48. Zhang R, et al. Transcriptional regulation of BMP2 expression by the PTH-CREB signaling pathway in osteoblasts. PLoS One. 2011;6(6):e20780.

49. Ghosh-Choudhury N, et al. Autoregulation of mouse BMP-2 gene transcription is directed by the proximal promoter element. Biochem Biophys Res Commun. 2001;286(1):101-108.

50. Zhang R, et al. Transcriptional regulation of BMP2 expression by the PTH-CREB signaling pathway in osteoblasts. PLoS One. 2011;6(6):e20780.

51. Wozney JM, et al. Novel regulators of bone formation: molecular clones and activities. Science. 1988;242(4885):1528-1534.

52. Celeste AJ, et al. Identification of transforming growth factor beta family members present in bone-inductive protein purified from bovine bone. Proc Natl Acad Sci U S A. 1990;87(24):9843-9847.

53. Turgeman G, et al. Systemically administered rhBMP-2 promotes MSC activity and reverses bone and cartilage loss in osteopenic mice. J Cell Biochem. 2002;86(3):461-474.

54. Israel DI, et al. Heterodimeric bone morphogenetic proteins show enhanced activity in vitro and in vivo. Growth Factors. 1996;13(3-4):291-300.

55. Borovecki F, Jelic M, Grgurevic L, Sampath KT, Bosukonda D, Vukicevic S. Bone morphogenetic protein-7 from serum of pregnant mice is available to the fetus through placental transfer during early stages of development. Nephron Exp Nephrol. 2004;97(1):e26-32.

56. Kung AL, et al. Gene dose-dependent control of hematopoiesis and hematologic tumor suppression by CBP. Genes Dev. 2000;14(3):272-277.

57. Giles RH, Peters DJ, Breuning MH. Conjunction dysfunction: $\mathrm{CBP} / \mathrm{p} 300$ in human disease. Trends Genet. 1998;14(5):178-183.

58. Roelfsema JH, et al. Genetic heterogeneity in Rubinstein-Taybi syndrome: mutations in both the CBP and EP300 genes cause disease. Am J Hum Genet. 2005;76(4):572-580.

59. Hennekam RC, et al. Deletion at chromosome 16 p13.3 as a cause of Rubinstein-Taybi syndrome: clinical aspects. Am J Hum Genet. 1993; 52(2):255-262.

60. Choi KY, et al. Runx2 regulates FGF2-induced Bmp2 expression during cranial bone development. Dev Dyn. 2005;233(1):115-121.

61. Bialek P, et al. A twist code determines the onset of osteoblast differentiation. Dev Cell. 2004; $6(3): 423-435$.

62. Ferron $\mathrm{M}$, et al. Insulin signaling in osteoblasts integrates bone remodeling and energy metabolism. Cell. 2010;142(2):296-308.

63. Gaide O, Schneider P. Permanent correction of an inherited ectodermal dysplasia with recombinant EDA. Nat Med. 2003;9(5):614-618.

64. Mundlos S, et al. Mutations involving the transcription factor CBFA1 cause cleidocranial dysplasia. Cell. 1997;89(5):773-779.

65. McLeod MJ. Differential staining of cartilage and bone in whole mouse fetuses by alcian blue and alizarin red S. Teratology. 1980;22(3):299-301.

66. Hildebrand T, Ruegsegger P. Quantification of bone microarchitecture with the Structure Model Index. Comput Methods Biomech Biomed Engin. 1997; 1(1):15-23.

67. Greenblatt MB, et al. The p38 MAPK pathway is essential for skeletogenesis and bone homeostasis in mice. J Clin Invest. 2010;120(7):2457-2473.

68. Jones DC, Wein MN, Oukka M, Hofstaetter JG, Glimcher MJ, Glimcher LH. Regulation of adult bone mass by the zinc finger adapter protein Schnurri-3. Science. 2006;312(5777):1223-1227. 